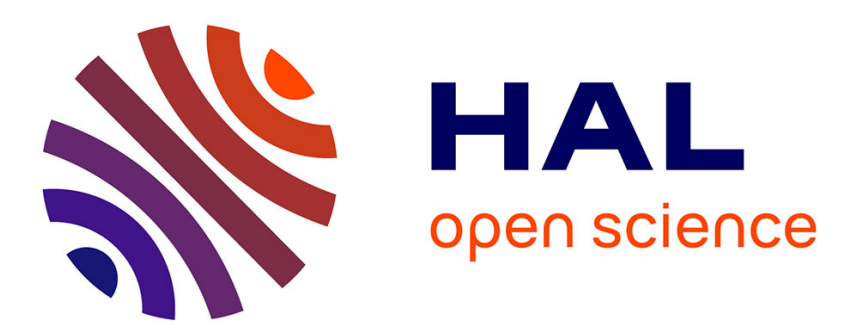

\title{
A relaxation scheme for hybrid modelling of gas-particle flows
}

Kateryna Dorogan, Mathieu Guingo, Jean-Marc Hérard, Jean-Pierre Minier

\section{To cite this version:}

Kateryna Dorogan, Mathieu Guingo, Jean-Marc Hérard, Jean-Pierre Minier. A relaxation scheme for hybrid modelling of gas-particle flows. Computers and Fluids, 2012, 70, pp.148_165. 10.1016/j.compfluid.2012.09.015 . hal-01265317

\section{HAL Id: hal-01265317 https://hal.science/hal-01265317}

Submitted on 1 Feb 2016

HAL is a multi-disciplinary open access archive for the deposit and dissemination of scientific research documents, whether they are published or not. The documents may come from teaching and research institutions in France or abroad, or from public or private research centers.
L'archive ouverte pluridisciplinaire HAL, est destinée au dépôt et à la diffusion de documents scientifiques de niveau recherche, publiés ou non, émanant des établissements d'enseignement et de recherche français ou étrangers, des laboratoires publics ou privés. 


\title{
A relaxation scheme for hybrid modelling of gas-particle flows
}

\author{
K. Dorogan ${ }^{1,2}$, M. Guingo ${ }^{1}$, J.M. Hérard ${ }^{1, *}$, J.P. Minier ${ }^{1}$ \\ ${ }^{1}$ Department of Fluid Dynamics, Power Generation and Environment, \\ EDF R\&D, 6 quai Watier, 78400 Chatou, France \\ ${ }^{2}$ LATP, CMI, 39 rue Joliot Curie, 13453 Marseille, France
}

\begin{abstract}
This paper aims at proposing a relaxation scheme that allows to obtain stable approximations for a system of partial differential equations which governs the evolution of the void fraction and the mean velocity in the particle phase of two-phase flows. This system involves the divergence of a particle kinetic tensor, which is provided by a Lagrangian code and whose components are not smooth. The simulation algorithm is based on the combined use of upwinding and relaxation techniques. The main properties of the method are given, together with the Finite Volume Godunov scheme and this approach is compared to an analogous one that was developed earlier. Some measured rates of convergence in $L^{1}$-norm are provided, for a particular choice of the kinetic tensor. To complete the picture, we present some numerical results obtained when non-smooth external data are provided to the system.
\end{abstract}

\section{Introduction}

In the present paper, we are interested in the numerical solution of a system of partial differential equations arising from a hybrid modelling of polydispersed turbulent two-phase flows $[10,25,26]$. The general framework of the hybrid modelling is recalled in Appendix C, which explains in particular a coupling of Eulerian and Lagrangian descriptions introduced for the modelling of the dispersed phase of gas-particle two-phase flows. The main difficulty of such a coupling consists in the treatment of non-smooth data provided by the Lagrangian part of the model in the divergence operator in the partial differential equations.

In $d$ space dimensions, we denote by $\mathbf{x}$ the space variable, $t$ the time, $\rho(t, \mathbf{x})$ the mean particle density, $\mathbf{u}(t, \mathbf{x}), \mathbf{U}(t, \mathbf{x}) \in \mathbb{R}^{d}$ the instantaneous and the mean particle velocities, $\mathbf{R}^{L}(t, \mathbf{x}) \in \mathbb{R}^{d \otimes d}$ the particle kinetic tensor, which can also be written as $R_{i j}^{L}=\left\langle u_{i}^{\prime} u_{j}^{\prime}\right\rangle^{L}$ with $i, j=1, \ldots, d$ and $\mathbf{u}^{\prime}(t, \mathbf{x})=\mathbf{u}(t, \mathbf{x})-\mathbf{U}(t, \mathbf{x})$ the fluctuation of the particle velocity. The following system, which is deduced from the Lagrangian stochastic model (Appendix $\mathrm{C}$ ), describes the time evolution of the first-order mean particle quantities:

$$
\left\{\begin{array}{l}
\partial_{t} \rho+\partial_{x_{j}}\left(\rho U_{j}\right)=0, \quad t>0, \quad \mathbf{x} \in \mathbb{R}^{d}, \\
\partial_{t}\left(\rho U_{i}\right)+\partial_{x_{j}}\left(\rho U_{i} U_{j}\right)+\partial_{x_{j}}\left(\rho R_{i j}^{L}\right)=\rho g_{i}+\rho\left\langle U_{r, i}^{L} / \tau_{p}^{U},\right\rangle,
\end{array}\right.
$$

where the superscript " $L$ " refers to variables computed using a Lagrangian description which, therefore, represent external non-smooth data. The right-hand side terms in the second equation in (1) describe the gravity and the drag forces, where $\mathbf{U}_{r}^{L}$ stands for the local relative velocity of the fluid and the dispersed phases, and $\tau_{p}^{U}$ is the particle velocity relaxation time scale (the time necessary for a particle to adjust to the fluid velocity).

*Corresponding author. Tel.: +3313087 70 37; E-mail address: jean-marc.herard@edf.fr 
Obviously, due to ensemble averaging, these equations are not closed since the correlations of the fluctuating particle velocities $R_{i j}^{L}(t, \mathbf{x})$ appear explicitly. From analogous situations arising with Reynolds averaging in single phase flow, these stresses have an important influence on the nature of the convective subset. This fact suggests that they should be handled with great care. It is also important to emphasize that $R_{i j}^{L}(t, \mathbf{x})$ is not smooth since it contains the statistical error that may be a cause of instabilities. Furthermore, we know that treating this convective term as a source term is not allowed (see [6] for a similar situation). The need to overcome these difficulties has led us to search for a method which would be stable during unsteady simulations with non-smooth external data.

In order to compute stable approximations of solutions of (1), a first approach has been proposed in $[10,26,27]$. This approach, which will be referred to as A1, relies on the combined use of relaxation-type techniques $[1,4,5,9,12,13,28,35,36,38]$ and upwinding schemes $[14,19,22,23,29,30]$ and was first introduced in [25] in order to predict single-phase turbulent flows [32]. In the latter approach, an extended hyperbolic system was introduced in a natural way, following what is done for previous associated Eulerian models (see [2, 3, 6, 21]). Though they contain non-conservative contributions, which are active in genuinely non linear fields, these "relaxation" models enable to obtain stable results. However, they involve a priori approximate jump conditions that may inhibit the convergence to the correct shock solutions of original system (1). This has motivated a new approach relying on a hyperbolic relaxation model, where all fields are linearly degenerate and, therefore, jump conditions are uniquely defined. This second approach introduced in this work will be referred to as A2. Both approaches A1 and A2 will be presented and compared in terms of convergence, stability and accuracy.

Before beginning to discuss our new approach of simulation, it is worth noting that we compute approximate solutions of (1) using a splitting strategy, where we treat first the corresponding homogeneous system:

$$
\left\{\begin{array}{l}
\partial_{t} \rho+\partial_{x_{j}}\left(\rho U_{j}\right)=0, \quad t>0, \quad \mathbf{x} \in \mathbb{R}^{d}, \\
\partial_{t}\left(\rho U_{i}\right)+\partial_{x_{j}}\left(\rho U_{i} U_{j}\right)+\partial_{x_{j}}\left(\rho R_{i j}^{L}\right)=0,
\end{array}\right.
$$

and then account for source terms through the second step:

$$
\left\{\begin{array}{l}
\partial_{t} \rho=0, \\
\partial_{t}\left(\rho U_{i}\right)=\rho g_{i}+\rho\left\langle U_{r, i}^{L} / \tau_{p}^{U}\right\rangle .
\end{array}\right.
$$

The paper is organized as follows. We give first the main guidelines of the relaxation procedure in the one-dimensional space. This will be detailed in section 2. The numerical scheme will be given in section 3. Section 4 will be devoted to the assessment of the numerical approach and to the comparison with the previous approach (A1) $[10,26,27]$, while focusing on analytical solutions in the particular case $\rho R^{L}=\pi(\rho)$ that refers to the barotropic Euler equations for gas dynamics. A quantitative analysis of the $L^{1}$ error norm will also be given. Some results given in appendices will complete the whole approach. Appendix B will focus on the energy control through both approaches (A1) and (A2) whatever $R$ is. The last appendix will provide some two-dimensional numerical results of coupling in realistic situations, where the kinetic tensor $R_{i j}^{L}$ is provided at each time step by an external computation using a Lagrangian description.

\section{Relaxation approach}

\section{$2.1 \quad$ Guidelines}

We are looking for a method that would ensure stable unsteady approximations of the solution to system (2) in one space dimension:

$$
\left\{\begin{array}{l}
\partial_{t} \rho+\partial_{x}(\rho U)=0, \quad t>0, \quad x \in \mathbb{R}, \\
\partial_{t}(\rho U)+\partial_{x}\left(\rho U^{2}+\rho R^{L}\right)=0 .
\end{array}\right.
$$


It is necessary to recall first that, since the kinetic tensor $\mathbf{R}^{\mathbf{L}}(\mathbf{t}, \mathbf{x})$ is non-smooth external data which, in practical coupled computations with the Lagrangian code, is not a function of variables $(\rho, \rho U)$, we are formally interested in finding "discontinuous" solutions of the problem. This may happen even if $R^{L}(t, x)=c s t>0$. These properties have led us to focus on Riemann solvers, these being particularly well suited for the simulation of problems with discontinuous solutions.

In this approach, we suggest that an Eulerian transport equation for the stresses be solved along with the above-mentioned mean particle quantities. The only purpose of the particle stress equation is to lead to a system of equations with a complete and meaningful description of the physical wave properties. The stress field will in fact be provided by the Lagrangian part of the overall model at each time step, but by keeping a (redundant) transport equation for the stresses, we will mimic the correct wave propagation within the Eulerian part, and thus will help to stabilize numerical approximations of the density and of the particle velocity.

To approximate the solutions of Eqs. (1) in the one-dimensional space, we introduce an augmented state vector $W(t, x)=(\rho, \rho U, \rho R)^{t}$ and a generalized relaxation system:

$$
\left\{\begin{array}{l}
\partial_{t} \rho+\partial_{x}(\rho U)=0 \\
\partial_{t}(\rho U)+\partial_{x}\left(\rho U^{2}+\rho R\right)=0, \quad t>0, \quad x \in \mathbb{R}, \\
\partial_{t}(\rho R)+\mathcal{A}\left(W, \partial_{x} W\right)=\rho\left(R^{L}-R\right) / \tau_{p}^{R} .
\end{array}\right.
$$

In order to guarantee the consistency of the new system with the original one at the discrete level, a relaxation time scale $\tau_{p}^{R}$ is introduced, and when this parameter is set formally to zero, the new system is consistent with the initial one.

Furthermore, we want to find an appropriate form of the operator $\mathcal{A}\left(W, \partial_{x} W\right)$, which describes the evolution of the fluctuating particle velocities, so that some important physical properties were respected. More precisely, we enforce the following features:

- (C1) we wish to define a hyperbolic system of equations. Hyperbolicity is one clue that the physical reality is correctly modelled and moreover it guarantees some inner stability of the solutions. For this reason, it seems crucial to preserve the property of hyperbolicity, which characterizes the original system.

- (C2) we intend to have a system characterized by linearly degenerated (LD) fields. This feature avoids imposing approximate jump conditions. Actually, we note that in the conservative system (2), jump relations are defined in a unique way. This essentially differs from the strategy introduced in [25, 26, 27], where the relaxation system is chosen to be close to the physics and hence contains non-conservative terms active in genuinely non-linear (GNL) fields.

- (C3) the system should preserve the positivity of the density distribution:

$$
\rho(x, t) \geq 0 .
$$

This property is expected in order to yield a physically meaningful solution.

- (C4) The relaxation system should be close enough to (A1) (introduced in [26, 27] and recalled below in sec. 2.2) that represents a relevant physical choice.

In the general case, the realizability of the kinetic tensor might also be required, which means that the associated quadratic form remains positive:

$$
n_{i} R_{i j} n_{j} \geq 0 \quad \forall n_{i}, n_{j}(i, j=1, \ldots, d) .
$$

In the one-dimensional case it amounts to ensure $R(t, x)>0$ for all $t>0, x \in \mathbb{R}$. Actually, the relaxation approach will guarantee this property at the discrete level, at each time step, in all cells. 


\subsection{System construction}

A preliminary study has revealed that conservative forms of the third equation in relaxation system (5) are not acceptable because they generally result in degenerate hyperbolic systems (eigenvectors no longer span the whole space). Thus, as the most appropriate and the simplest candidate for the larger system in one space dimension, we suggest the following:

$$
\left\{\begin{array}{l}
\partial_{t} \rho+\partial_{x}(\rho U)=0, \\
\partial_{t}(\rho U)+\partial_{x}\left(\rho U^{2}\right)+\partial_{x}(\rho R)=0, \quad t>0, x \in \mathbb{R} \\
\partial_{t}(\rho R)+U \partial_{x}(\rho R)+\Psi \partial_{x} U=\rho\left(R^{L}-R\right) / \tau_{p}^{R},
\end{array}\right.
$$

where $\tau_{p}^{R}$ denotes some small relaxation time scale. Here, the choice of the evolution operator is reduced to the choice of the function:

$$
\Psi(\rho, R)>0
$$

which influences considerably the characteristics of the whole system. In the remainder of this section, we are interested mainly by the properties of the homogeneous system corresponding to (7):

$$
\left\{\begin{array}{l}
\partial_{t} \rho+\partial_{x}(\rho U)=0, \\
\partial_{t}(\rho U)+\partial_{x}\left(\rho U^{2}\right)+\partial_{x}(\rho R)=0, \quad t>0, x \in \mathbb{R} \\
\partial_{t}(\rho R)+U \partial_{x}(\rho R)+\Psi \partial_{x} U=0 .
\end{array}\right.
$$

Since the convective part of the model is not in conservative form, we define a non-conservative variable $Z=(\rho, U, \rho R)^{t}$. Then, for smooth solutions, system (9) can be rewritten in the following condensed form:

$$
\partial_{t} Z+A(Z) \partial_{x} Z=0, \quad t>0, x \in \mathbb{R}
$$

with the system matrix

$$
A(Z)=\left(\begin{array}{ccc}
U & \rho & 0 \\
0 & U & \vartheta \\
0 & \Psi & U
\end{array}\right)
$$

where $\vartheta(x, t)=1 / \rho(x, t)$ is the specific volume.

Property 1. For all $Z$ in the phase space $\Omega=\left\{(\rho, U, \rho R)^{t} \in \mathbb{R}^{3}\right.$ such that $\left.\rho>0\right\}$, system (10) admits three real distinct eigenvalues:

$$
\begin{aligned}
& \lambda_{1}=U-c \\
& \lambda_{2}=U \\
& \lambda_{3}=U+c
\end{aligned}
$$

with $\rho c^{2}=\Psi>0$. The corresponding right eigenvectors are given by:

$$
\vec{r}_{1}=\left(\begin{array}{c}
\rho \\
-c \\
\rho c^{2}
\end{array}\right) \quad \vec{r}_{2}=\left(\begin{array}{l}
1 \\
0 \\
0
\end{array}\right) \quad \vec{r}_{3}=\left(\begin{array}{c}
\rho \\
c \\
\rho c^{2}
\end{array}\right)
$$

and span $\mathbb{R}^{3}$. Thus, the system (10) is hyperbolic on $\Omega$ (unless vacuum occurs in the solution).

A natural choice for $\Psi$ arising from $R_{i j}$ model in one-dimensional space was given in [27]: $\Psi=3 \rho R$. This is referred to as approach (A1). 


\section{Property 2.}

(A1): If we assume that $\Psi=3 \rho R$, then system (9) admits two GNL fields and one LD field;

(A2): If we assume that $\Psi=a_{0}^{2} \vartheta$ with constant $a_{0} \in \mathbb{R}_{+}^{*}$, then system (9) admits three LD fields.

In fact, for calculated eigenvalues $\lambda_{k}$ and eigenvectors $\vec{r}_{k}(k=1,2,3)$ of $A(Z)$ and for any function $\Psi(\rho, R)>0$ we have:

$$
\begin{aligned}
& \frac{\partial \lambda_{1}}{\partial Z} \cdot \vec{r}_{1}=\frac{\partial(U-c)}{\partial Z} \cdot \vec{r}_{1}=-\rho \frac{\partial c}{\partial \rho}-c-\rho c^{2} \frac{\partial c}{\partial(\rho R)}=-\frac{\partial \lambda_{3}}{\partial Z} \cdot \vec{r}_{3}, \\
& \frac{\partial \lambda_{2}}{\partial Z} \cdot \vec{r}_{2}=\frac{\partial U}{\partial Z} \cdot \vec{r}_{2}=0, \quad \Rightarrow \quad 2-\text { field is LD. }
\end{aligned}
$$

- If we take $\Psi=3 \rho R$ then $c^{2}=\Psi / \rho=3 R$ and the 1 and the 3 fields are GNL, because

$$
\frac{\partial \lambda_{1}}{\partial Z} \cdot \vec{r}_{1}=-\frac{\partial \lambda_{3}}{\partial Z} \cdot \vec{r}_{3}=-2 c=\neq 0 .
$$

- It is also easily seen that for all function $\Psi=\Psi(\rho)=a_{0}^{2} \vartheta$ with constant $a_{0}$ the following equality is verified for $c=\sqrt{\Psi / \rho}=a_{0} \vartheta$ :

$$
\frac{\partial \lambda_{1}}{\partial Z} \cdot \vec{r}_{1}=-\frac{\partial \lambda_{3}}{\partial Z} \cdot \vec{r}_{3}=0 .
$$

This means that all the fields in this case are LD.

In order to ensure the link with the expression in (A1), in (A2) we fix $a_{0}=\sqrt{3 R_{0} \rho_{0}^{2}}$ (see Appendix A (part II)).

Remark 1. The form of the function $\Psi$ of the approach (A1) is "natural", but it requires introducing approximate jump conditions (see [26], [27]). The choice of $\Psi$ associated with (A2) ensures the uniqueness of the jump conditions and results in the relaxation system that corresponds exactly to the system introduced for the simulation of barotropic Euler equations, when $\rho R^{L}=\pi(\rho)$. When restricting to (A2) and though equation (9.3) is slightly different from the formulations presented for instance in [4] and [9] for barotropic Euler equations (see Appendix A too), relaxation systems are equivalent for smooth solutions and the underlying idea is the same.

Remark 2. System (7) ensures the positivity of the density function. It follows from the mass continuity equation that when $\forall x, \rho(t=0, x)=\rho_{0}>0$, the density values may never become negative in $[0, T]$ as soon as $U, \partial_{x} U \in L^{\infty}\left(\mathbb{R}_{x} \times[0, T]\right)$.

Remark 3. Some energy estimates for relaxation systems corresponding to (A1) and (A2) are given in $\boldsymbol{A}$ ppendix $\boldsymbol{B}$, in a one-dimensional framework (and in $[18,16]$ in a $2 D$ framework). These should not be confused with entropy estimates for barotropic Euler equations for instance, such as those provided in [4], [35] and [9] among others.

In the remainder of this paper we will focus on the presentation of the approach (A2). A comparison of results obtained with both (A1) and (A2) will be provided and discussed in section 4 .

\subsection{Analytical solution of the Riemann problem}

Before going further on, we recall that the one dimensional Riemann problem corresponds to the initial value problem associated with the hyperbolic system (9) and discontinuous initial conditions:

$$
\left[\begin{array}{c}
\rho \\
U \\
\rho R
\end{array}\right]=\left[\begin{array}{c}
\rho_{l} \\
U_{l} \\
(\rho R)_{l}
\end{array}\right] \text { for } x \leq 0 \text { and }\left[\begin{array}{c}
\rho \\
U \\
\rho R
\end{array}\right]=\left[\begin{array}{c}
\rho_{r} \\
U_{r} \\
(\rho R)_{r}
\end{array}\right] \text { for } x>0 \text {. }
$$


In the remainder of the paper, we will use the following shortened notations:

$$
\begin{aligned}
& \bar{U}_{l r}=\frac{U_{l}+U_{r}}{2}, \quad[U]_{l}^{r}=\left(U_{r}-U_{l}\right), \quad[\rho R]_{l}^{r}=(\rho R)_{r}-(\rho R)_{l}, \\
& r_{1}=\left\{\begin{array}{l}
\max \left(\frac{-[U]_{l}^{r} \pm \sqrt{\left([U]_{l}^{r}\right)^{2}+8 \vartheta_{l}[\rho R]_{l}^{r}}}{4 \vartheta_{l}}\right), \quad \text { if } \quad\left([U]_{l}^{r}\right)^{2}+8 \vartheta_{l}[\rho R]_{l}^{r} \geq 0 \\
0, \quad \text { otherwise }
\end{array}\right. \\
& r_{2}=\left\{\begin{array}{l}
\max \left(\frac{-[U]_{l}^{r} \pm \sqrt{\left([U]_{l}^{r}\right)^{2}-8 \vartheta_{r}[\rho R]_{l}^{r}}}{4 \vartheta_{r}}\right), \quad \text { if } \quad\left([U]_{l}^{r}\right)^{2}-8 \vartheta_{r}[\rho R]_{l}^{r} \geq 0 \\
0, \quad \text { otherwise }
\end{array}\right.
\end{aligned}
$$

Property 3 (Existence and Uniqueness of the solution of the Riemann problem for (A2)). Assume that initial conditions are physically relevant $\left(\rho_{l, r}>0\right.$ and $\left.R_{l, r}>0\right)$. Assume moreover that $a_{0}>a_{0}^{\min }=\max \left(r_{1}, r_{2}, 0\right) \geq 0$ with constant $a_{0}$ and $r_{1}, r_{2}$ given by (16), (17). Then the Riemann problem associated with the homogeneous part of (7) that is (9), initial conditions (14) and $\Psi=a_{0}^{2} \vartheta$, admits a unique solution $(t, x) \mapsto Z^{\text {exact }}\left(x / t ; Z_{l}, Z_{r}\right)$, composed of four constant states $Z_{l}, Z_{1}, Z_{2}, Z_{r}$ separated by three $L D$ waves. $Z_{1}$ and $Z_{2}$ are given by the following analytical expressions:

$$
\begin{gathered}
\vartheta_{1}=\vartheta_{l}+\frac{U_{r}-U_{l}}{2 a_{0}}-\frac{(\rho R)_{r}-(\rho R)_{l}}{2 a_{0}^{2}} \\
\vartheta_{2}=\vartheta_{r}+\frac{U_{r}-U_{l}}{2 a_{0}}+\frac{(\rho R)_{r}-(\rho R)_{l}}{2 a_{0}^{2}} \\
U_{1}=U_{2}=\frac{U_{l}+U_{r}}{2}-\frac{(\rho R)_{r}-(\rho R)_{l}}{2 a_{0}} \\
(\rho R)_{1}=(\rho R)_{2}=\frac{(\rho R)_{l}+(\rho R)_{r}}{2}-\frac{a_{0}\left(U_{r}-U_{l}\right)}{2} .
\end{gathered}
$$

A straightforward consequence is that $\vartheta_{1}, \vartheta_{2}>0$ and the waves are ordered $\left(\lambda_{1}<\lambda_{2}<\lambda_{3}\right)$.

A proof of this result can be found in [9] for instance, with a slightly different form of the relaxation system. The proof is obtained by construction and we recall it below. Let us consider only the solutions of the class of self-similar functions $(x, t) \mapsto Z(x / t)$, composed of constant states separated by three contact discontinuities (fig. 1):

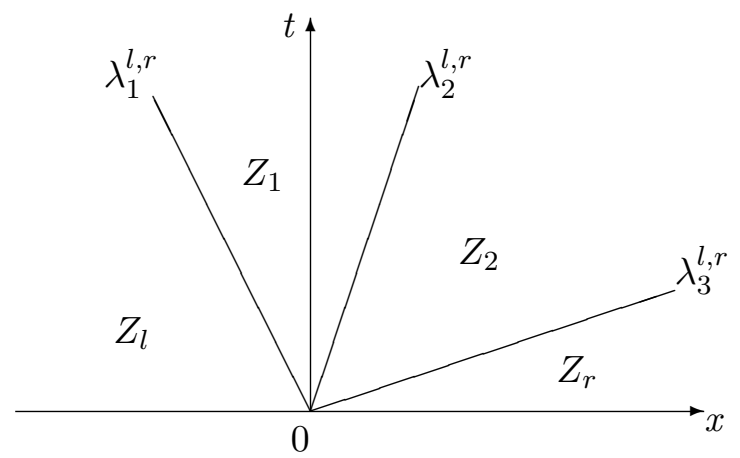

Figure 1: Sketch of the solution of the one-dimensional Riemann problem when $\lambda_{2}^{l, r}>0$.

Here, $\lambda_{1}^{l, r}=U_{l}-a_{0} \vartheta_{l}, \lambda_{2}^{l, r}=U_{1}=U_{2}=\bar{U}_{l r}-[\rho R]_{l}^{r} / 2 a_{0}, \lambda_{3}^{l, r}=U_{r}+a_{0} \vartheta_{r}$. 
Associated with each $k$-wave we have two $k$-Riemann Invariants, $I_{R}^{k}(k=1,2,3)$, which satisfy:

$$
\frac{\partial I_{R}^{k}}{\partial Z} \cdot \vec{r}_{k}=0
$$

These $k$-Riemann Invariants remain constant through the $k$-contact discontinuity. For the present 1-D problem, Riemann Invariants are given by the following expressions:

$$
\begin{aligned}
& I_{R}^{1}=\left\{U-c, \rho R+a_{0}^{2} \vartheta\right\} \\
& I_{R}^{2}=\{U, \rho R\} \\
& I_{R}^{3}=\left\{U+c, \rho R+a_{0}^{2} \vartheta\right\}
\end{aligned}
$$

Then, the structure of the solution of the Riemann problem can be written as:

1-wave

$$
\left\{\begin{array}{l}
U_{l}-c_{l}=U_{1}-c_{1} \\
(\rho R)_{l}+a_{0}^{2} \vartheta_{l}=(\rho R)_{1}+a_{0}^{2} \vartheta_{1}
\end{array}\right.
$$

2-wave

$$
\left\{\begin{array}{l}
U_{1}=U_{2} \\
(\rho R)_{1}=(\rho R)_{2}
\end{array}\right.
$$

3-wave

$$
\left\{\begin{array}{l}
U_{2}+c_{2}=U_{r}+c_{r} \\
(\rho R)_{2}+a_{0}^{2} \vartheta_{2}=(\rho R)_{r}+a_{0}^{2} \vartheta_{r}
\end{array}\right.
$$

We check at once that formulae (18), (19), (20) are the unique solutions of (22), (23), (24). Eventually, the constructed analytical solution to the Riemann problem associated with (9) and initial conditions (14) can be sketched as:

$$
(x, t) \mapsto Z^{\text {exact }}\left(x / t ; Z_{l}, Z_{r}\right) .
$$

For $a_{0}$ large enough, the positivity of $\vartheta_{1}, \vartheta_{2}$ is ensured for all initial conditions $(\rho, U, \rho R)_{l, r}$. At the same time, we would like to avoid $a_{0}$ being too large in order to preserve the accuracy of the scheme. Hence, we aim at determining an appropriate value of $a_{0}$. Using notations (15), we can rewrite the wave ordering condition $\lambda_{1}<\lambda_{2}<\lambda_{3}$ as follows:

$$
U_{l}-a_{0} \vartheta_{l}<\bar{U}_{l r}-\frac{1}{2 a_{0}}[\rho R]_{l}^{r}<U_{r}+a_{0} \vartheta_{r} .
$$

After some simple transformations, this leads us to the next system of square inequalities :

$$
\left\{\begin{array}{l}
2 \vartheta_{l} a_{0}^{2}+[U]_{l}^{r} a_{0}-[\rho R]_{l}^{r}>0 \\
2 \vartheta_{r} a_{0}^{2}+[U]_{l}^{r} a_{0}+[\rho R]_{l}^{r}>0
\end{array}\right.
$$

and we can see that imposing $\vartheta_{1}, \vartheta_{2}>0$ in (18) leads to the same constraints. The solution of (27) provides us with "natural" conditions to choose a physically relevant lower bound for $a_{0}$.

Remark 4. When $\rho R^{L}=\pi(\rho)$ with $\pi$ given, a further evaluation of a lower bound of the parameter $a_{0}$ needed in (A2) will be recalled in Appendix $\boldsymbol{A}$, relying on Whitham's condition. This condition guarantees some dissipation in the entropy balance equation (see [9]). In the general case, where $\rho R^{L}=\rho R^{L}(t, x)$, this condition cannot be directly applied (see Appendix A).

Remark 5. The positivity of the kinetic tensor in the intermediate states is not preserved for any initial condition $(\rho, U, \rho R)_{l, r}$. However, it is crucial to emphasize that at each time step the local values of $R=R^{L}$ are restored, thus the realizability is ensured owing to the instantaneous relaxation step. 
Remark 6. In the approach (A1) developed in [27], the positivity of the kinetic tensor is required to ensure the hyperbolicity property for the corresponding relaxation system and, at the same time, is preserved by the very construction of this system.

\section{Numerical approximation}

Following the relaxation technique described in section 2, we approximate the solutions of an initial value problem associated with original system (4) by those of extended system (7) with $\Psi=a_{0}^{2} \vartheta$. For simplicity of the notations, both problems can be rewritten in a condensed form:

$$
\left\{\begin{array}{l}
\partial_{t} W_{\phi}+\partial_{x} F_{\phi}\left(W_{\phi}, R^{L}\right)=0, \quad t>0, x \in \mathbb{R} \\
W_{\phi}(0, x)=W_{\phi, 0}(x) \text { and } R^{L}(0, x)=R_{0}^{L}(x)
\end{array}\right.
$$

for (4) with $W_{\phi}=(\rho, \rho U)^{t}, F_{\phi}\left(W_{\phi}, R^{L}\right)=\left(\rho U, \rho U^{2}+\rho R^{L}\right)^{t}$, and

$$
\left\{\begin{array}{l}
\partial_{t} W+\partial_{x} F(W)+G(W) \partial_{x} W=S\left(W, R^{L}\right), \quad t>0, x \in \mathbb{R} \\
W(0, x)=W_{0}(x)
\end{array}\right.
$$

for (7), where $W=(\rho, \rho U, \rho R)^{t}, W \in \Omega$ is the unknown vector with the corresponding physical "fluxes" and the source term $S\left(W, R^{L}\right)$ :

$$
F(W)=\left(\begin{array}{c}
\rho U \\
\rho U^{2}+\rho R \\
\rho U R
\end{array}\right), \quad G(W) \partial_{x} W=\left(\begin{array}{c}
0 \\
0 \\
\left(a_{0}^{2} \vartheta-\rho R\right) \partial_{x} U
\end{array}\right), \quad S\left(W, R^{L}\right)=\left(\begin{array}{c}
0 \\
0 \\
\rho\left(R^{L}-R\right) / \tau_{p}^{R}
\end{array}\right) .
$$

We consider a uniform grid given by cell centers $x_{i}=i h, i \in \mathbb{Z}$. Each cell is an interval $\mathcal{C}_{i}=\left[x_{i-1 / 2}, x_{i+1 / 2}\right]$ of length $h$, where the points $x_{i+1 / 2}=x_{i}+h / 2$ are called cell interfaces. The time is discretized with the time step $(\Delta t)^{n}$ such that $t^{n+1}=t^{n}+(\Delta t)^{n}, n \in \mathbb{N}$ are intermediate times. We assume the values of the approximate solution at time $t^{n}, x \in \mathbb{R} \mapsto W_{\Delta}\left(x, t^{n}\right) \in \Omega$, to be constant in each cell $\mathcal{C}_{i}, i \in \mathbb{Z}$, and denote them by $W_{i}^{n}$ :

$$
W_{\Delta}\left(x, t^{n}\right)=W_{i}^{n}, \quad \forall x \in \mathcal{C}_{i}, i \in \mathbb{Z}, n \in \mathbb{N} .
$$

Besides, using the initial condition $W_{0}(x)$ at $t=0$, we define the sequence $\left(W_{i}^{0}\right)_{i \in \mathbb{Z}}$ as the average of function $W_{0}(x)$ over the cell $i(i \in \mathbb{Z})$ :

$$
W_{i}^{0}=\frac{1}{h} \int_{x_{i-1 / 2}}^{x_{i+1 / 2}} W_{0}(x) d x, \quad \forall i \in \mathbb{Z}
$$

At $t=0, W_{\Delta}\left(x, t^{0}\right)$ is set at equilibrium which means that $(\rho R)_{i}^{0}=\left(\rho R^{L}\right)_{i}^{0}, i \in \mathbb{Z}$.

At each time step, the global algorithm which provides the approximations $W_{\Delta}\left(., t^{n+1}\right)$ from $W_{\Delta}\left(., t^{n}\right)$ can be written as follows:

- Initialization (at $\left.t^{n}\right)$ : As initial condition we take $W_{i}^{n}=\left(\rho_{i}^{n}, \rho_{i}^{n} U_{i}^{n}, \rho_{i}^{n} R_{i}^{L, n}\right)^{t}$;

- Evolution step $\left(t^{n} \rightarrow t^{n+1,-}\right)$ : approximation of the solution of the homogeneous system corresponding to (29) at $t^{n+1,-}=t^{n}+\Delta t^{n}$ with a Finite Volume scheme described in sec.3.1. This step computes the values $W_{i}^{n+1,-}$ from $W_{i}^{n}(i \in \mathbb{Z})$;

- Relaxation step $\left(t^{n+1,-} \rightarrow t^{n+1}\right)$ : accounts for the source term $S\left(W, R^{L}\right)$ by solving the differential equation $\partial_{t} W=S\left(W, R^{L}\right)$ at $t^{n+1}=t^{n}+\Delta t^{n}$ with the initial condition 
$W_{i}^{n+1,-}$ obtained at the previous stage. It traduces the return of the system to equilibrium assuming an instantaneous relaxation $\left(\tau_{p}^{R} \rightarrow 0\right)$ and gives the values $W_{i}^{n+1}, i \in \mathbb{Z}$ :

$$
\left\{\begin{array}{l}
\rho_{i}^{n+1}=\rho_{i}^{n+1,-} \\
(\rho U)_{i}^{n+1}=(\rho U)_{i}^{n+1,-} \\
(\rho R)_{i}^{n+1}=\rho_{i}^{n+1}\left(R^{L}\right)_{i}^{n+1} \Leftrightarrow R_{i}^{n+1}=\left(R^{L}\right)_{i}^{n+1} .
\end{array}\right.
$$

Finally, we retrieve $W_{\phi, i}^{n+1}=\left(\rho_{i}^{n+1},(\rho U)_{i}^{n+1}\right)^{t}, i \in \mathbb{Z}, n \geq 0$ that is an approximation of the solution to (28).

\subsection{Evolution step: Godunov scheme and interface Riemann solver}

The computations inside this step are made in terms of the conservative state vector $W=$ $(\rho, \rho U, \rho R)^{t}$. Therefore, we approximate solutions of the following Cauchy problem

$$
\left\{\begin{array}{l}
\partial_{t} W+\partial_{x} F(W)+G(W) \partial_{x} W=0, t \in\left[0,(\Delta t)^{n}\right], x \in \mathbb{R} \\
W(x, 0)=W_{\Delta}\left(x, t^{n}\right) .
\end{array}\right.
$$

Following properties 1, 2, this system is hyperbolic and admits three LD fields. Thus, the solution of the associated Riemann problem is uniquely defined. Since $x \mapsto W_{\Delta}\left(x, t^{n}\right)$ is piecewise constant, the solution to system (34) is obtained by solving the Riemann problem at each cell interface $x_{i+1 / 2}, i \in \mathbb{Z}$. To be consistent, the time step $(\Delta t)^{n}, n \in \mathbb{N}$ should be limited in such a way that the waves emanating from an interface do not interact with waves created at the adjacent interfaces during the time step. This leads to the CFL condition:

$$
\frac{(\Delta t)^{n}}{h} \max _{W}\left(\left|\lambda_{k}(W)\right|, \quad k=1,2,3\right) \leq C F L<\frac{1}{2}, n \in \mathbb{N} .
$$

More precisely, for $\left.\left.(x, t) \in\left[x_{i}, x_{i+1}\right] \times\right] 0, \Delta t\right]$ we have

$$
W_{\Delta}(x, t)=W^{\text {exact }}\left(\frac{x-x_{i+1 / 2}}{t} ; W_{i}^{n}, W_{i+1}^{n}\right), i \in \mathbb{Z} .
$$

where $(x, t) \mapsto W^{\text {exact }}\left(x / t ; W_{l}, W_{r}\right)$ is the self-similar solution of the Riemann problem

$$
\left\{\begin{array}{l}
\partial_{t} W+\partial_{x} F(W)+G(W) \partial_{x} W=0, \\
W(x, 0)= \begin{cases}W_{l} & \text { if } x<0 \\
W_{r} & \text { if } x>0\end{cases}
\end{array}\right.
$$

given by property 3 . Then, we can write

$$
W^{\text {exact }}\left(x / t ; W_{i}^{n}, W_{i+1}^{n}\right)= \begin{cases}W_{i}^{n}, & \text { if } \frac{x}{t} \leq \lambda_{1, i+1 / 2}, \\ W_{1, i+1 / 2}^{n}, & \text { if } \lambda_{1, i+1 / 2}<\frac{x}{t}<\lambda_{2, i+1 / 2}, \\ W_{2, i+1 / 2}^{n}, & \text { if } \lambda_{2, i+1 / 2}<\frac{x}{t}<\lambda_{3, i+1 / 2}, \\ W_{i+1}^{n}, & \text { if } \frac{x}{t} \geq \lambda_{3, i+1 / 2},\end{cases}
$$

where $W_{i}^{n}, W_{i+1}^{n}$ are averaged cell values and $W_{1, i+1 / 2}^{n}, W_{2, i+1 / 2}^{n}$ are intermediate states in the solution of the Riemann problem at the interface $i+1 / 2$ (Fig. 2).

In the following, we use this exact solution $W_{\Delta}(x, t)$ to construct the approximation $W_{i}^{n+1,-}, i \in$ $\mathbb{Z}$ with a Godunov finite volume scheme:

$$
h\left(W_{i}^{n+1,-}-W_{i}^{n}\right)+\Delta t^{n}\left(\mathcal{F}_{i+\frac{1}{2}}^{n, \text { God }}-\mathcal{F}_{i-\frac{1}{2}}^{n, \text { God }}\right)+\Delta t^{n} \mathcal{H}_{i}^{n}=0, \quad i \in \mathbb{Z}, n \geq 0,
$$

where $\mathcal{F}_{i+\frac{1}{2}}^{n, \text { God }}=\mathcal{F}\left(W_{i}^{n}, W_{i+1}^{n}\right)$ is the exact Godunov flux through the interface $i+1 / 2$ :

$$
\mathcal{F}_{i+\frac{1}{2}}^{n, \text { God }}=F\left(W^{\text {exact }}\left(0 ; W_{i}^{n}, W_{i+1}^{n}\right)\right),
$$




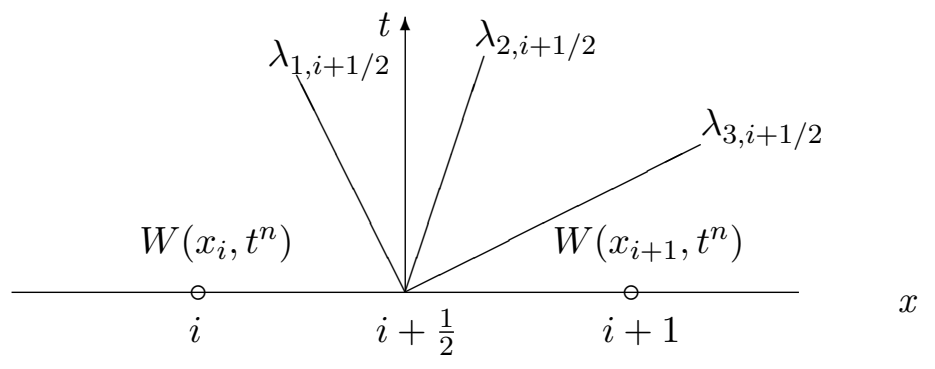

Figure 2: Sketch of a part of the grid

and $\mathcal{H}_{i}^{n}$ is an approximation of the non-conservative contribution. If we denote for the sake of simplicity $W_{i+1 / 2}^{*}=W^{\text {exact }}\left(0 ; W_{i}^{n}, W_{i+1}^{n}\right), i \in \mathbb{Z}$, the term $\mathcal{H}_{i}^{n}$ is given by:

$$
\mathcal{H}_{i}^{n}=\left(\begin{array}{c}
0 \\
0 \\
\frac{1}{2}\left(\left(a_{0}^{2} \vartheta\right)_{i-\frac{1}{2}}^{*}+\left(a_{0}^{2} \vartheta\right)_{i+\frac{1}{2}}^{*}-(\rho R)_{i-\frac{1}{2}}^{*}-(\rho R)_{i+\frac{1}{2}}^{*}\right)\left(U_{i+\frac{1}{2}}^{*}-U_{i-\frac{1}{2}}^{*}\right)
\end{array}\right),
$$

where the locally constant values $\left(a_{0}^{2}\right)_{i+1 / 2}^{n}$ are computed at each $t^{n}, n \in \mathbb{N}$ at each cell interface $i+1 / 2, i \in \mathbb{Z}$ according to the wave ordering condition and, when possible, Whitham's condition (see Appendix A for further details).

The present algorithm gives us the piecewise constant values $Z_{\Delta}\left(x, t^{n+1,-}\right)$ that are approximations averaged on each cell $\mathcal{C}_{i}, i \in \mathbb{Z}$.

Remark 7 (Positivity of cell values of the density). Assume that $\left|\lambda_{\max }\right| \Delta t / h<1 / 2$ and that the parameter $a_{0}$ guarantees the positivity of the density in the intermediate states defined by (18): $\vartheta_{1, i+1 / 2}, \vartheta_{2, i+1 / 2}>0, i \in \mathbb{Z}$. Then the evolution step (39) preserves the positivity of the cell values of the density and the whole algorithm computes $\rho_{i}^{n+1} \geq 0$ (since the relaxation step (33) preserves cell densities).

The proof being classical is not recalled here.

\section{Numerical results}

In order to verify the approach (A2) we consider in the first subsections below some test cases where :

$$
\rho R^{L}=\pi(\rho),
$$

and thus, analytical solutions are known. Some theoretical results for (A2) (seen as a "true" relaxation technique for the barotropic Euler equations) can be found in this case in $[4,9]$ for instance and references called therein. Such a closure $\rho R^{L}=\pi(\rho)$ allows to measure the error of approximation and to verify the convergence towards the correct solution in practice. By the way, we will compare results obtained with both (A2) and (A1). The numerical scheme for approach (A1) has been proposed in [27] and is not recalled here. It relies on a different "relaxation" system (see section 2.2) and on the use of the VFRoe-ncv scheme [8]. In order to emphasize the main differences between (A1) and (A2) we present numerical tests on shock tubes for the most difficult configurations, such as vacuum occurrence, strong shocks and rarefaction waves including sonic points.

In the second part, some results obtained with specific analytic kinetic tensor $R^{L}$ will be presented. 


\subsection{Algorithm verification}

In this part of the paper, we will assume the following "closure" relation:

$$
\rho R^{L}=\pi(\rho)=S_{0} \rho^{\gamma}
$$

with constant entropy $S_{0}=10^{5}$ and $\gamma=3$. This value of $\gamma$ corresponds to the underlying isentropic case arising in [27], [6]. Thus

$$
R_{i}^{L, n}=S_{0}\left(\rho_{i}^{n}\right)^{\gamma-1}
$$

within each cell $i$, and we can compute the exact solution of the one-dimensional Riemann problem associated with the homogeneous analogue of (1) in all cases.

Initial conditions refer to different 1D Riemann problems. The computational domain consists of a one-dimensional tube placed in $[-0.5,0.5]$ with a membrane in the middle, which separates two different fluid states at the beginning of the computation $(t=0)$. All meshes used to solve these Riemann problems are regular. The time step is in agreement with the CFL condition and the CFL number is equal to $1 / 2$. For a qualitative study, we present profiles for the density, the velocity, the pressure and the flow rate. From a quantitative point of view, numerical convergence curves, at a given time, are represented by plotting the logarithm of the L1-norm of the error as a function of the logarithm of the mesh size. We recall that the expected rate of convergence for the isentropic one-dimensional Euler equations is equal to 1, due to the fact that the system only involves two GNL fields. The grids that have been used contain between $N=10^{2}$ and $N=10^{5}$ cells.

In order to stabilize the numerical approximations at each $t^{n}, n \in \mathbb{N}$ and at each cell interface $i+1 / 2, i \in \mathbb{Z}$, we impose the (locally constant) values $\left(a_{0}\right)_{i+1 / 2}^{n}$ to satisfy the condition:

$$
\left(a_{0}\right)_{i+1 / 2}^{n} \geq \max \left(\left(a_{0}\right)_{i+1 / 2}^{\lambda, n},\left(a_{0}\right)_{i+1 / 2}^{W, n}\right)
$$

where $a_{0}^{\lambda}$ and $a_{0}^{W}$ are defined in Appendix $\boldsymbol{A}$ for all $n \in \mathbb{N}, i \in \mathbb{Z}$.

\subsubsection{Supersonic shock tube}

We study first a supersonic case, using the following initial conditions:

$$
\begin{gathered}
\rho_{l}=1, \quad \rho_{r}=0.35, \\
U_{l}=100, \quad U_{r}=290 .
\end{gathered}
$$

The exact solution is composed of a supersonic 1 - rarefaction wave and a right going 2 - shock wave, Fig. 4. From the Fig. 5 we may conclude that approximations obtained with the two schemes converge towards the correct solution with the same rate of convergence (which is about 0.85 and compares well with rates given in $[7,11]$ ). Moreover, both methods provide almost the same accuracy for a given mesh size. In this case, the 1-rarefaction wave contains a sonic point. The VFRoe-ncv scheme used in approach (A1) requires an entropy correction at sonic points [24]; otherwise a wrong shock wave develops. On the other hand, the relaxation scheme (A2) exhibits the correct behaviour in the rarefaction wave without any entropy correction.

\subsubsection{Symmetrical double shock wave}

This case is interesting in order to compare the stability of both schemes around a wall boundary, when the normal component of the velocity of the fluid close to the boundary is positive. We consider the initial conditions:

$$
\rho_{l}=\rho_{r}=1
$$




$$
U_{l}=-U_{r}=10^{3} .
$$

The exact solution is composed of a 1 - shock wave and a 2 - shock wave. The numerical rate of convergence for both the density and the velocity is approximately 1 for both methods, which is in agreement with results obtained in [7] (see Fig. 6, 7). Moreover, the accuracy for the two schemes is almost the same for a given mesh size. Eventually, we emphasize that the relaxation approach (A2) exhibits a better stability since (A1) induces tiny - stable - oscillations near the shocks (that do not inhibit the convergence). Actually, this characterizes problems with a very high initial kinetic energy, even when the CFL number is such that waves do not interact. It has been shown that approximations obtained with a CFL number equal to 0.9 are also convergent in that case for (A2). In fact, the limit of stability of approach (A2) in the present configuration is reached for $U_{l}=-U_{r}=10^{4}$, where stable approximations are still available, but tiny oscillations appear (Fig. 8). On the other hand, approach (A1) is no longer suitable to tackle such a problem.

Remark 8. This test case is essential since it provides some numerical evidence that both approximate solutions provided by methods (A1) and (A2) converge to the correct shock solutions of original system (4). This result was not obvious for the relaxation system corresponding to approach (A2), but it was even less expected for (A1), where approximate jump conditions have been introduced in the "relaxation" system.

\subsubsection{Symmetrical double rarefaction wave}

We now examine a symmetrical double rarefaction wave. This one is the counterpart of the previous test, when the inlet normal component of the velocity is negative. Initial conditions are the following:

$$
\begin{gathered}
\rho_{l}=\rho_{r}=1, \\
U_{l}=-U_{r}=-100 .
\end{gathered}
$$

Two symmetric rarefaction waves develop when $U_{r}$ is positive. We have plotted the approximations of the solution in Fig. 10. The error obviously vanishes as the mesh size tends towards zero and the numerical rates of convergence are still close to 0.85 (Fig. 11).

\subsubsection{Symmetrical double rarefaction wave with vacuum occurrence}

Here, we study a more complex case where the speeds of rarefaction waves are so large that it leads to a vacuum occurrence in the solution. Before going further on, we recall that for isentropic Euler equations with a perfect gas equation of state, a vacuum may occur only when

$$
U_{r}-U_{l}>\frac{2}{\gamma-1}\left(c_{\phi, r}+c_{\phi, l}\right),
$$

where $c_{\phi}$ is the celerity associated with system (4) when $\rho R^{L}=\pi(\rho)=S_{0} \rho^{\gamma}$. Assuming the initial conditions:

$$
\begin{gathered}
\rho_{l}=\rho_{r}=1, \\
U_{l}=-U_{r}<0, \\
c_{\phi, l}=c_{\phi, r}=\sqrt{S_{0} \gamma \rho_{r}^{\gamma-1},}
\end{gathered}
$$

we conclude that the vacuum may occur when

$$
\frac{U_{r}}{c_{\phi, r}}>\frac{2}{\gamma-1} .
$$

If we take $U_{l}=-U_{r}=-1000$, relation (53) is satisfied. The exact solution is composed of two symmetric rarefaction waves. The intermediate state exhibits a vacuum zone where the velocity is not defined but the flow rate and the density are zero. When focusing on $(\rho, Q)$, both approximations obtained with (A1), (A2) converge towards the correct solution with vanishing $h$. A glance at profiles in Fig. 12 enables to check that both schemes give almost the same accuracy. Numerical rates of convergence in Fig. 13 are close to 0.7 (see also [7], [11], [20]). 


\subsubsection{Supersonic rarefaction wave}

This case is somewhat equivalent to a dam break with shallow-water equations. One initial condition (on the right) is such that the density is very small:

$$
\begin{gathered}
\rho_{l}=1, \quad \rho_{r}=10^{-7} \\
U_{l}=0, \quad U_{r}=U_{l}+\frac{2 c_{\phi, l}}{\gamma-1}\left(1-\frac{c_{\phi, r}}{c_{\phi, l}}\right) .
\end{gathered}
$$

Here, a supersonic 1 - rarefaction wave is followed by a "ghost" 2 - shock wave. Once more, we check that approximations converge towards the correct solution when the mesh is refined (Fig. 14). The rate of convergence is slightly higher than in the previous test case (about 0.82, Fig. 15). This suggests that the true vacuum zone actually pollutes the approximations. We note a small loss of monotonicity at the end of the rarefaction wave. Actually, this drawback vanishes with a mesh refinement, and therefore, the positivity of cell values of the density is preserved. As mentionned before, an important issue here is that the relaxation scheme (A2) does not need any entropy correction.

\subsection{Numerical results for (A2) with a noisy kinetic tensor}

We focus now on some numerical test cases when a noisy kinetic tensor is plugged in the system of equations (4). For that purpose, we apply a noise to the values of $R^{L}$, which refer to the isentropic case studied in section 4.1, at each time step in the cells that belong to the region $x \in[-0.25,0.25]$ as follows:

$$
R_{i}^{L, n}=S_{0}\left(\rho_{i}^{n}\right)^{\gamma-1}(1+\operatorname{rms}(0.5-\operatorname{rand}(0,1))), i \in \mathbb{Z}, n \in \mathbb{N},
$$

where $r m s$ stands for the noise intensity and rand (random numbers generator) allows to manage the noise amplitude.

We choose the initial conditions of a subsonic shock tube problem:

$$
\begin{gathered}
\rho_{l}=1, \quad \rho_{r}=0.24, \\
U_{l}=-10, \quad U_{r}=-282 .
\end{gathered}
$$

We fix the final time $T$, such that the boundary of the domain is not yet attained by the travelling waves. In order to estimate the stability of numerical approximations in such a case, we analyse their evolution when the mesh is refined: $N=10^{2}, 10^{3}, 10^{4}, 5 \times 10^{4}, 10^{5}$ cells. The comparison displayed in Fig. 3 shows that the noise is not diminishing when the mesh is refined, and that approximations do not converge towards some smooth solution. Moreover, we note that the $L^{1}$ norm of the difference between the approximations with $r m s=0.1$ and $r m s=0$ tends to be constant when $h$ tends to zero [17]. 

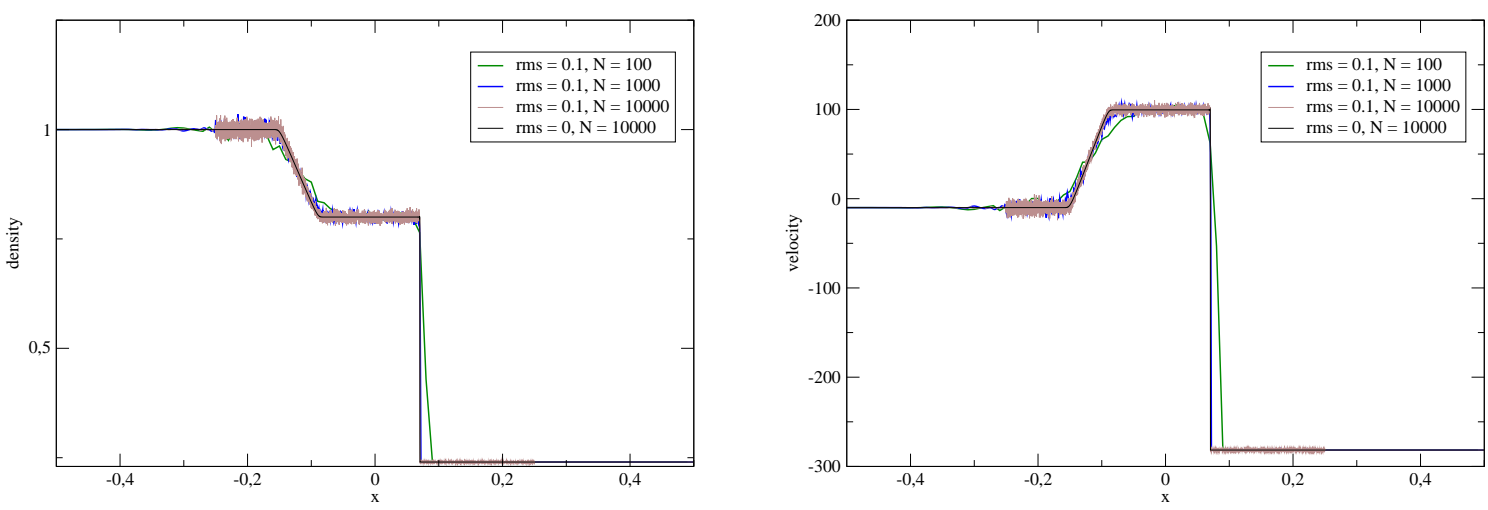

Figure 3: Subsonic shock tube: density and velocity approximations

\section{Conclusion}

We have introduced in this paper a new approach (A2) in order to tackle approximations of solutions to (1) in the one-dimensional case. This method is quite similar to (A1) introduced in $[10,26,27]$ but it no longer requires the definition of approximate jump conditions since the relaxation system admits only linearly degenerated fields. The approach (A2) has been validated by computing exact solutions in a specific case, where $R^{L}=R^{L}(\rho)$, and associated errors. Numerical results have also been compared with those provided by approach (A1). On the whole, we would like to note that:

- both methods (A1) and (A2) guarantee a correct convergence of approximations, even when shocks arise in the solution. This was foreseen to happen, due to the conservative form of the schemes associated with (A1) and (A2) for the basic two conservative equations in (1);

- both methods can handle vacuum occurrence. This is crucial for practical hybrid computations.

We also emphasize that approach (A1), which relies on the approximate Godunov solver VFRoe-ncv, requires an entropy correction at sonic points in rarefaction waves, whereas approach (A2) does not. Moreover, (A2) may handle stronger shock waves.

The reference [17] shows several numerical experiments which have been achieved while plugging noisy kinetic tensor in the governing set of partial differential equations (4). A sample result can be found in sec. 4.2. One may conclude from [17] that approaches (A1), (A2) enable stable computations, which means that a mesh refinement is always possible, whatever the noise level. Moreover, for a given noise, the difference between approximations obtained with or without noise varies linearly with respect to the noise intensity and disturbances due to the noisy region are smoothed by the numerical scheme and tend to disappear out of this region (see [17]).

Eventually, we note that the present relaxation approach has been extended to a two-dimensional framework. This work is presented roughly in [18] and in detail in [15, 16]. A two-dimensional coupled simulation in a realistic configuration is presented in Appendix C.

Acknowledgments:

The first author receives a financial support through an EDF-CIFRE contract 203/2009. Computational facilities were provided by EDF. We would like to thank Thierry Gallouet who accepted to read the initial manuscript. 

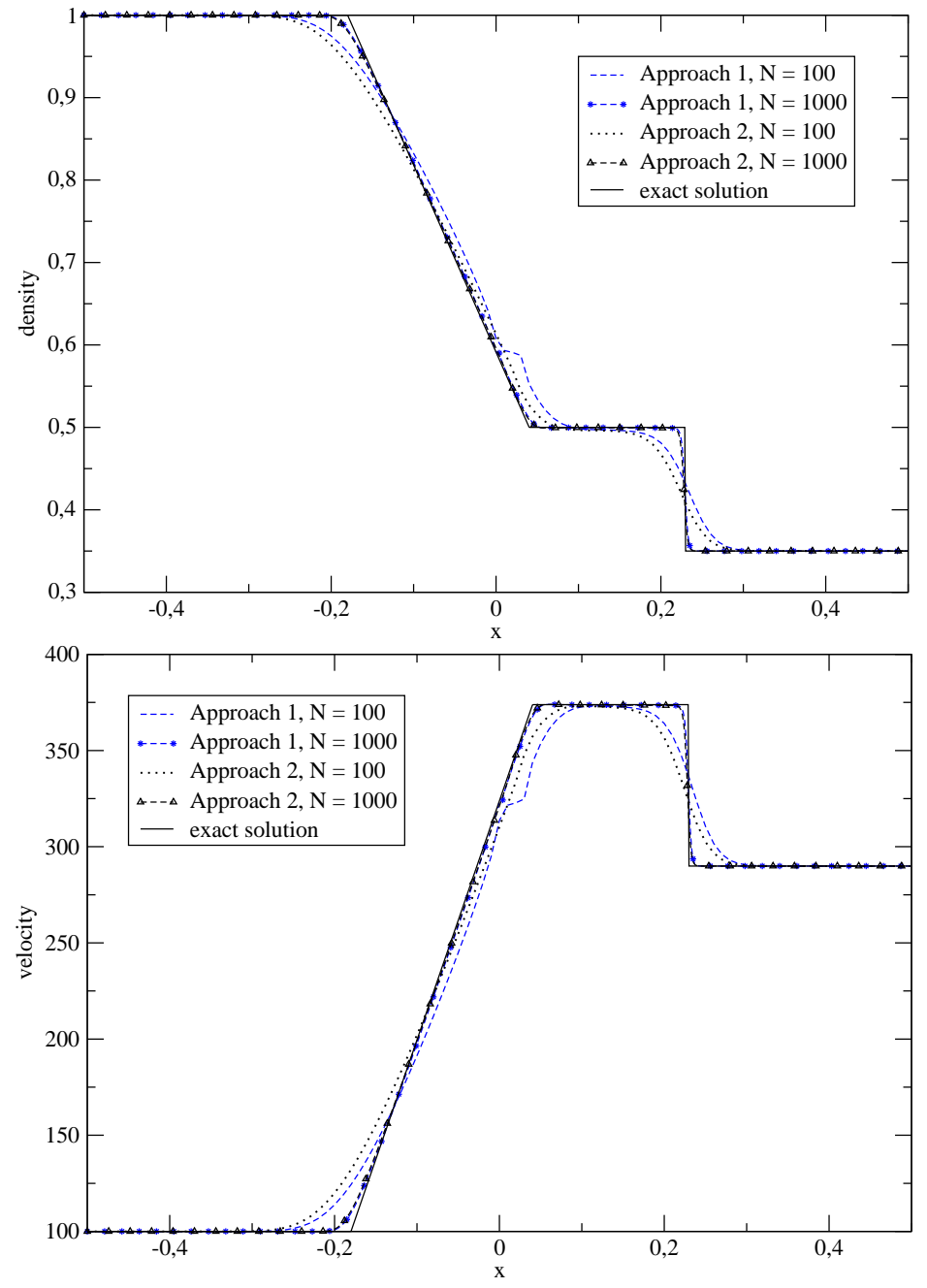

Figure 4: Supersonic shock tube: comparison of density and velocity solutions
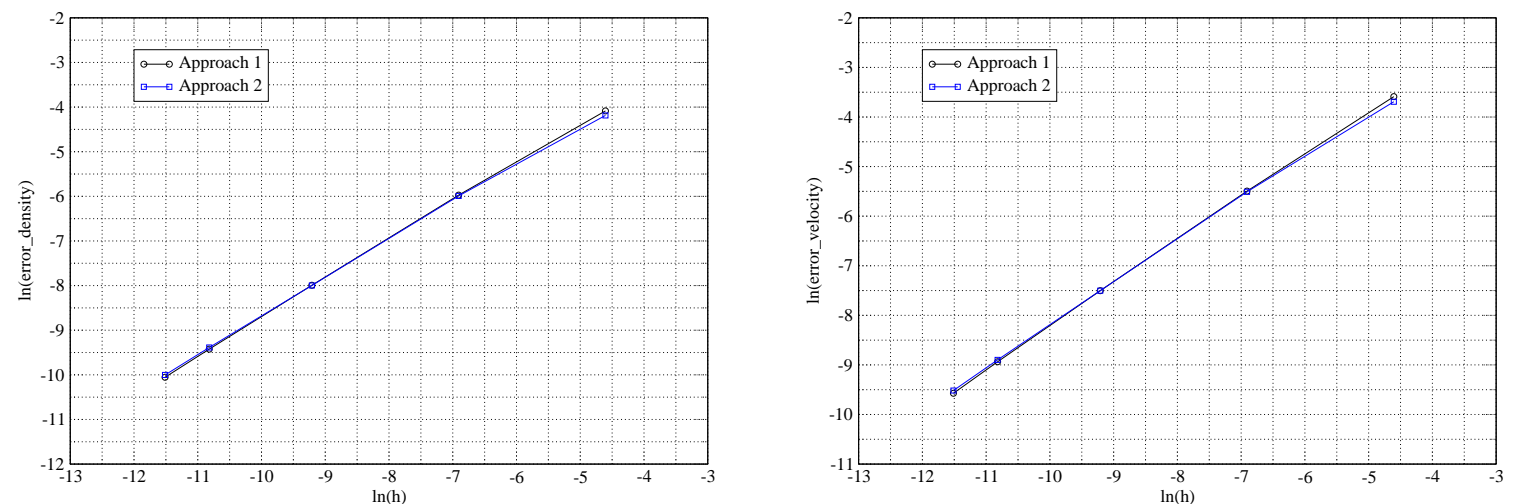

Figure 5: L1 convergence curves for supersonic shock tube: density (left), velocity (right). Coarser mesh: 100 cells; finer mesh: 100000 cells 

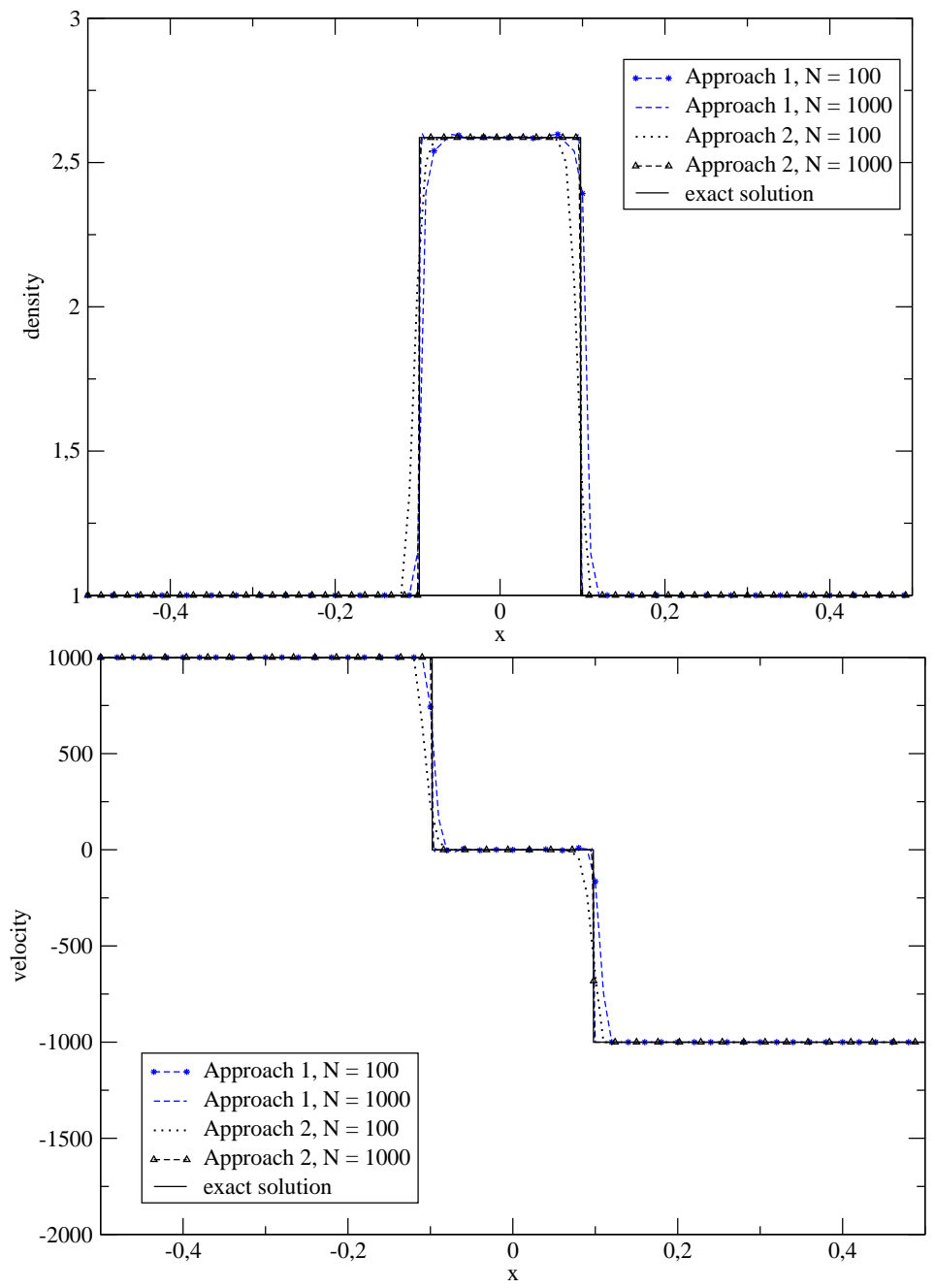

Figure 6: Symmetric double shock: comparison of density and velocity solutions
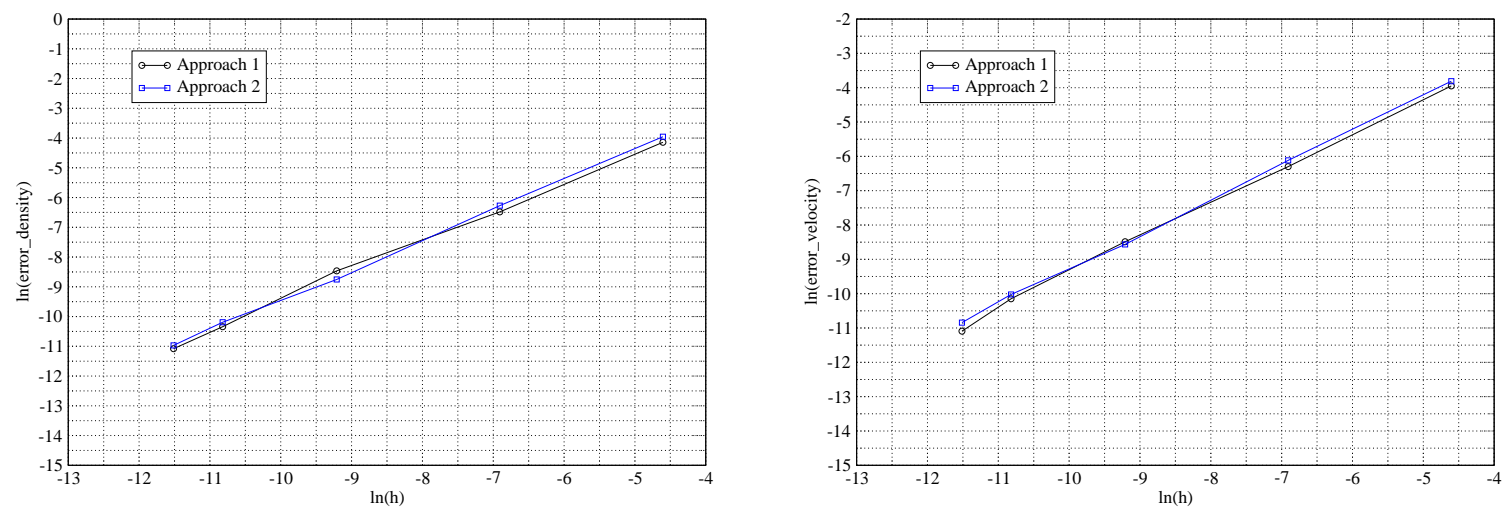

Figure 7: L1 convergence curves for symmetric double shock: density (left), velocity (right). Coarser mesh: 100 cells; finer mesh: 100000 cells 

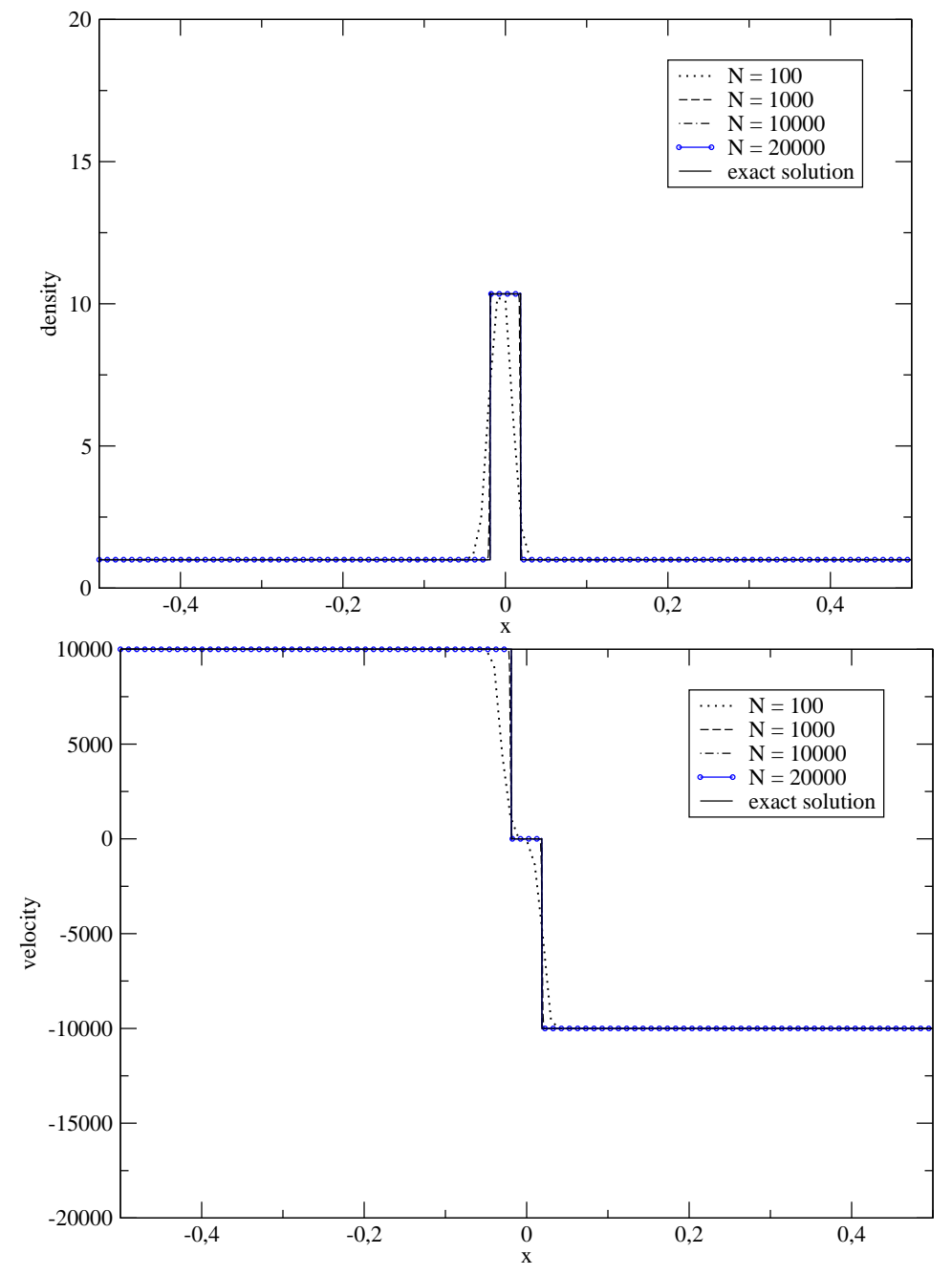

Figure 8: Symmetric double shock: density and velocity solutions with approach A2

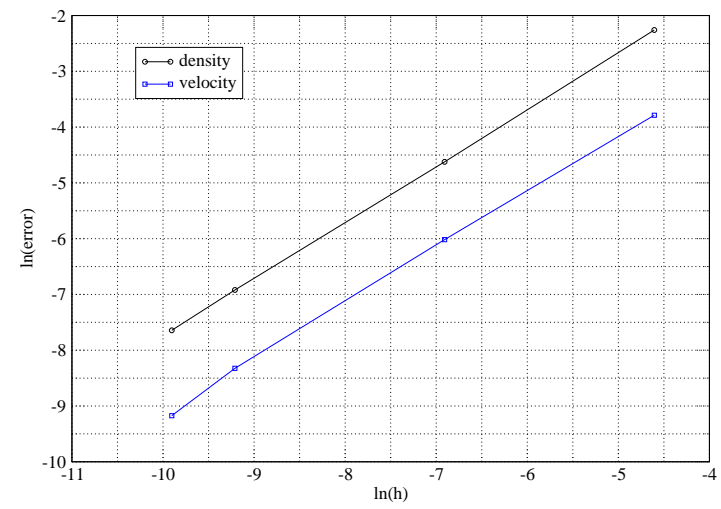

Figure 9: L1 convergence curves for symmetric double shock using approach A2. Coarser mesh: 100 cells; finer mesh: 100000 cells 

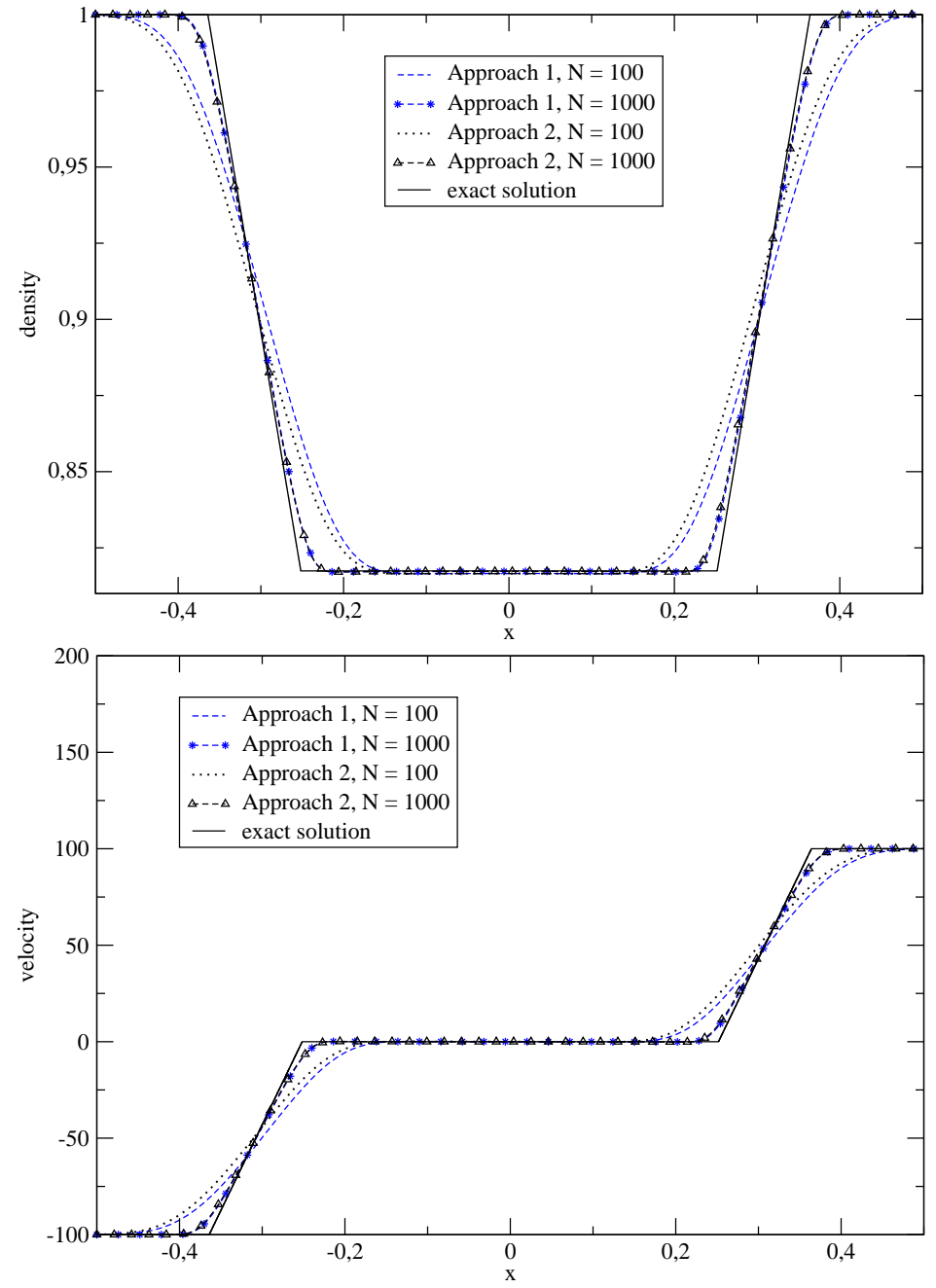

Figure 10: Symmetric double rarefaction: comparison of density and velocity solutions
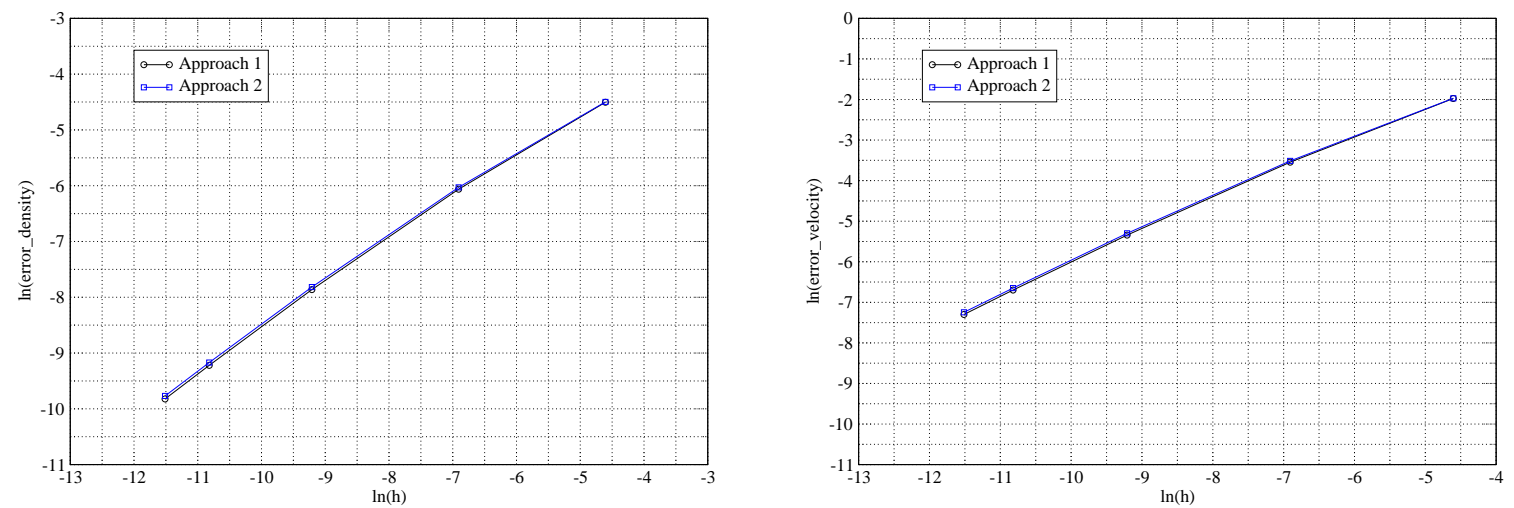

Figure 11: L1 convergence curves for symmetric double rarefaction: density (left), velocity (right). Coarser mesh: 100 cells; finer mesh: 100000 cells 

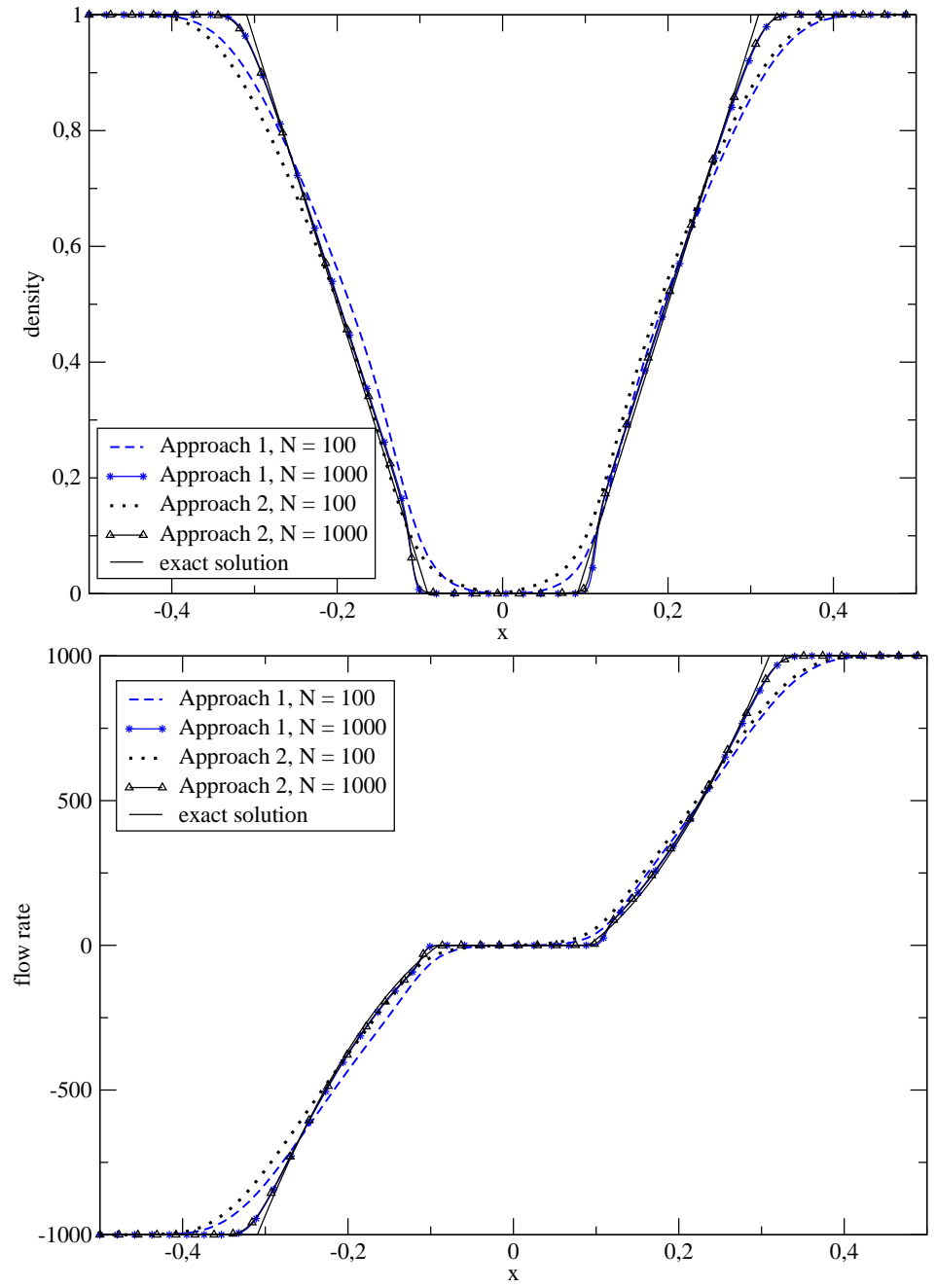

Figure 12: Symmetric double rarefaction with vacuum occurence: comparison of density and flow rate solutions
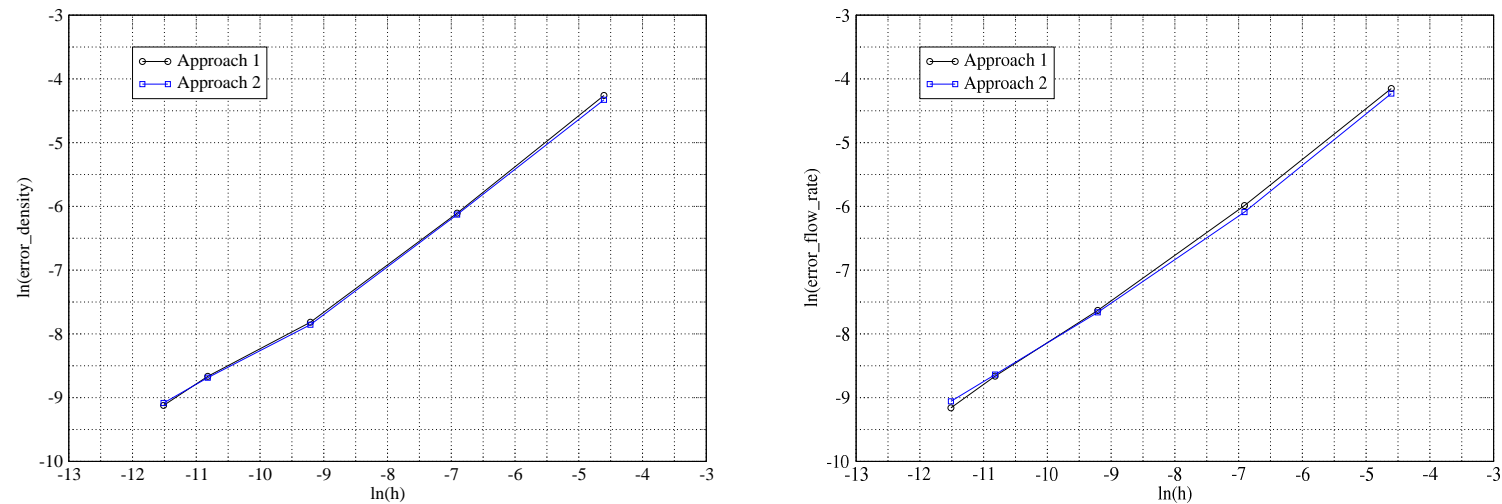

Figure 13: L1 convergence curves for symmetric double rarefaction with vacuum: density (left), flow rate (right). Coarser mesh: 100 cells; finer mesh: 100000 cells 

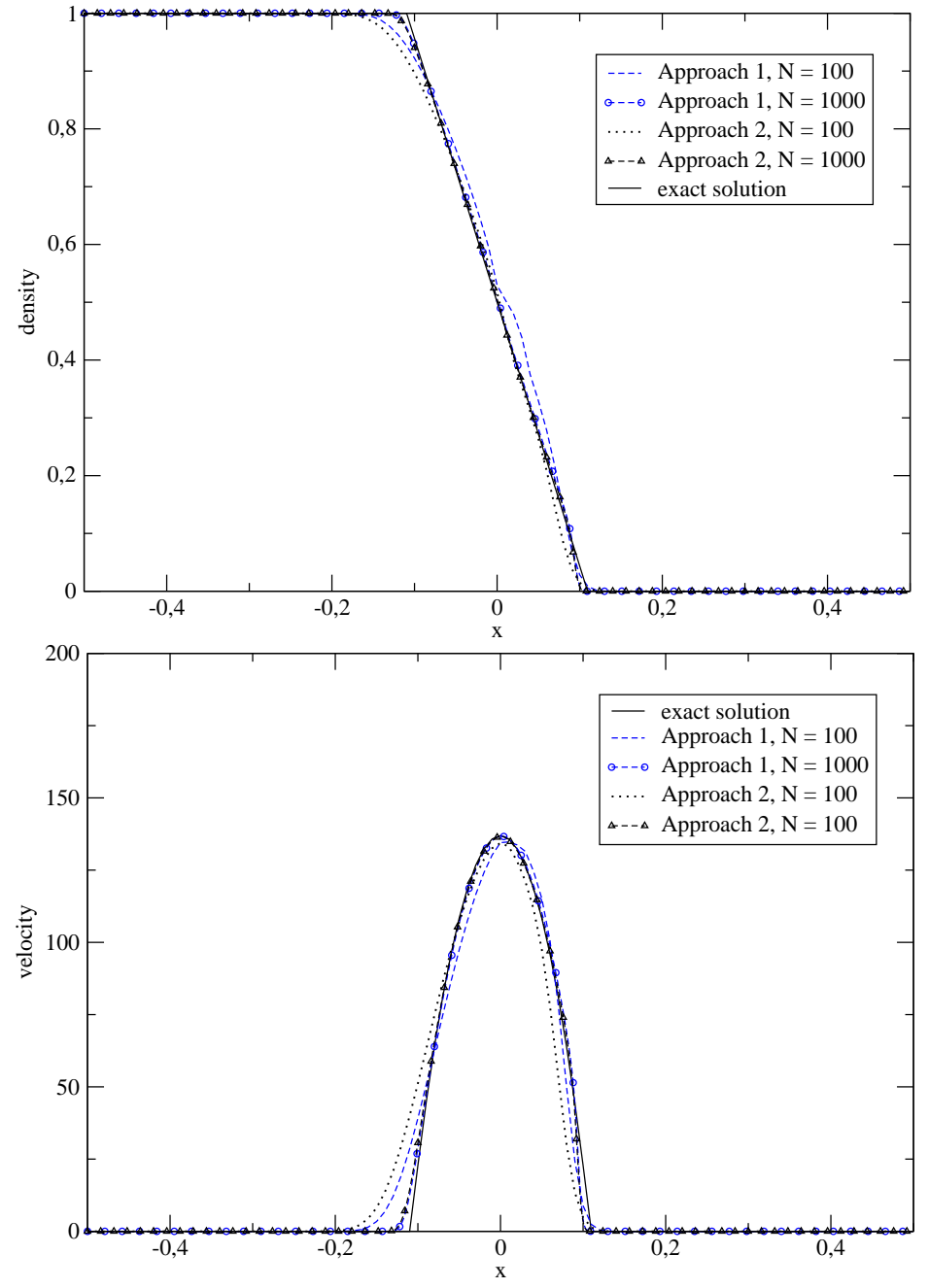

Figure 14: 1-supersonic rarefaction wave: comparison of density and velocity solutions
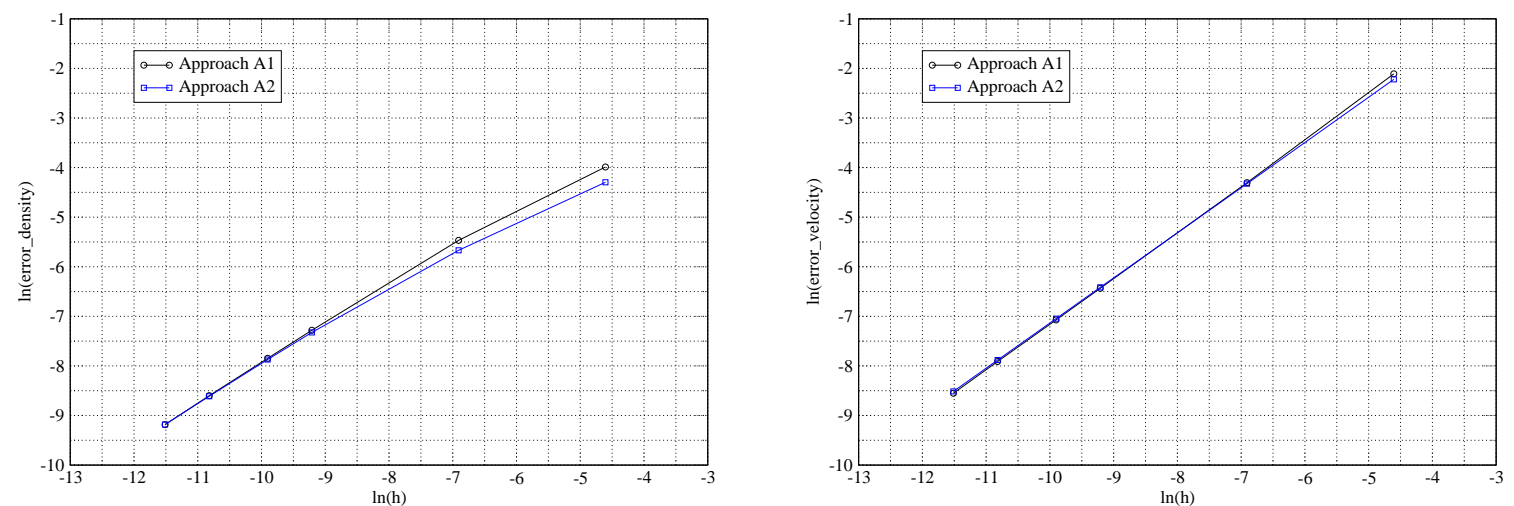

Figure 15: L1 convergence curve for 1-supersonic rarefaction wave: density (left), velocity (right). Coarser mesh: 100 cells; finer mesh: 100000 cells 


\section{Appendix A. $a_{0}$ parameter evaluation}

We consider two constant states $Z_{l}, Z_{r}$ that refer to the Riemann problem (9), (14) with $\Psi=a_{0}^{2} \vartheta$ and such that: $R_{l, r}>0$ and $\rho_{l, r}>0$. To find a suitable value of $a_{0}$ associated with this problem (that is the value that allows to find a compromise between the stability and the accuracy of numerical approximations) the following two conditions are imposed:

\section{- Wave ordering condition}

As it has been shown in sec. 2.3, the wave ordering condition

$$
\lambda_{1}(Z)<\lambda_{2}(Z)<\lambda_{3}(Z)
$$

ensures the positivity of $\vartheta_{1}, \vartheta_{2}$ and is used to get a lower bound for $a_{0}$. With shortened notations

$$
\bar{x}_{l r}=\frac{x_{l}+x_{r}}{2}, \quad[x]_{l}^{r}=\left(x_{r}-x_{l}\right)
$$

condition (58) leads us to the next system of inequalities:

$$
\left\{\begin{array}{l}
2 \vartheta_{l} a_{0}^{2}+[U]_{l}^{r} a_{0}-[\rho R]_{l}^{r}>0 \\
2 \vartheta_{r} a_{0}^{2}+[U]_{l}^{r} a_{0}+[\rho R]_{l}^{r}>0
\end{array}\right.
$$

with discriminants expressed by

$$
\begin{aligned}
& \Delta_{1}=\left([U]_{l}^{r}\right)^{2}+8 \vartheta_{l}[\rho R]_{l}^{r} \\
& \Delta_{2}=\left([U]_{l}^{r}\right)^{2}-8 \vartheta_{r}[\rho R]_{l}^{r} .
\end{aligned}
$$

Then, if $\Delta_{1} \geq 0$ we have

$$
\left(a_{0}\right)_{1,2}=\frac{-[U]_{l}^{r} \pm \sqrt{\Delta_{1}}}{4 \vartheta_{l}}
$$

When $\Delta_{2} \geq 0$, we find

$$
\left(a_{0}\right)_{3,4}=\frac{-[U]_{l}^{r} \pm \sqrt{\Delta_{2}}}{4 \vartheta_{r}}
$$

Finally, the whole algorithm for computing $a_{0}$ under the wave ordering condition can be summarized as follows.

\section{Algorithm:}

We take an initial value for the parameter $\varepsilon$ :

$$
\varepsilon=\left(3 R_{0}\right)^{1 / 2} \rho_{0} \quad \text { with } \quad R_{0}=\bar{R}_{l r}, \quad \rho_{0}=\bar{\rho}_{l r}
$$

If $\Delta_{1} \geq 0$ and $\Delta_{2} \geq 0$ then $a_{0}=\max \left\{\varepsilon,\left(a_{0}\right)_{i}\right\}, i=1,2,3,4$.

If $\Delta_{1} \geq 0$ and $\Delta_{2}<0$ then $a_{0}=\max \left\{\varepsilon,\left(a_{0}\right)_{i}\right\}, i=1,2$.

If $\Delta_{1}<0$ and $\Delta_{2} \geq 0$ then $a_{0}=\max \left\{\varepsilon,\left(a_{0}\right)_{i}\right\}, i=3,4$.

If $\Delta_{1}<0$ and $\Delta_{2}<0$ then $a_{0}=\varepsilon$.

Let us denote the lower bound obtained for $a_{0}$ at this stage by $a_{0}^{\lambda}$.

\section{- Whitham's condition}

- Following for instance [9], in order to obtain stable approximations of the barotropic Euler equations

$$
\left\{\begin{array}{l}
\partial_{t} \rho+\partial_{x}(\rho U)=0, \quad t>0, \quad x \in \mathbb{R} \\
\partial_{t}(\rho U)+\partial_{x}\left(\rho U^{2}\right)+\partial_{x} p(\tau)=0
\end{array}\right.
$$


where $\rho$ is the density, $U$ is the velocity, $\tau(t, x)=1 / \rho(t, x)$ is the specific volume, and $p$ is the pressure law, one may introduce a relaxation system of the form $(\lambda>0)$ :

$$
\left\{\begin{array}{l}
\partial_{t} \rho+\partial_{x}(\rho U)=0, \quad t>0, \quad x \in \mathbb{R} \\
\partial_{t}(\rho U)+\partial_{x}\left(\rho U^{2}\right)+\partial_{x} \pi=0 \\
\partial_{t}(\rho \mathcal{T})+\partial_{x}(\rho \mathcal{T} U)=\lambda \rho(\tau-\mathcal{T})
\end{array}\right.
$$

with the so-called relaxed pressure:

$$
\pi(\mathcal{T}, \tau)=p(\mathcal{T})+a_{0}^{2}(\mathcal{T}-\tau)
$$

which is expected to tend to $p(\tau)$ when $\mathcal{T} \rightarrow \tau$. Setting $\varepsilon(\tau): \varepsilon(\tau)=-p^{\prime}(\tau)$ and restricting to smooth solutions, the governing equation of the relaxation entropy

$$
\rho \Sigma=\frac{\rho U^{2}}{2}+\rho \varepsilon(\mathcal{T})+\rho \frac{\pi^{2}-p^{2}(\mathcal{T})}{2 a_{0}^{2}},
$$

is given by (see [9]):

$$
\partial_{t}(\rho \Sigma)+\partial_{x}(\rho \Sigma U+\pi U)=-\lambda \rho\left(a_{0}^{2}+p^{\prime}(\mathcal{T})\right)(\mathcal{T}-\tau)^{2} .
$$

Thus, the relaxation entropy is dissipated by the relaxation procedure if

$$
a_{0}^{2}>-p^{\prime}(\mathcal{T}), \text { for all realisable }(\tau, U, \mathcal{T})
$$

At the discrete level, the explicit numerical algorithm corresponding to formulation (63) only requires at each time $t=t^{n}$ the knowledge of

$$
\left\{\begin{array}{l}
\mathcal{T}^{n}=\tau^{n}=1 / \rho^{n} \\
\pi^{n}=p\left(\mathcal{T}^{n}\right)=p\left(\tau^{n}\right)=p^{n}
\end{array}\right.
$$

Hence, the explicit form of the function $p(\mathcal{T})$ is not compulsory, only the cell values $p^{n}$ are needed. However, condition $(65)$ requires an estimation of $p^{\prime}(\mathcal{T})$, which is unknown if $p(\mathcal{T})$ is unknown. Thus, if $p(\mathcal{T})$ is unknown, this entropy dissipation principle cannot be used in practice to compute a lower bound for $a_{0}^{2}$.

- In our framework, the correspondence can be made between the present system (62) and equations (4) setting $p\left(\tau, R^{L}\right)=R^{L} / \tau$, but a lower bound of $a_{0}^{2}$ cannot be obtained on the basis of (64), (65), which no longer make sense.

Thus, in order to ensure the numerical stability of the method at a discrete level, we introduce a local estimate:

$$
\frac{\Delta p}{\Delta \tau}=\frac{\Delta_{l, r}\left(R^{L} / \tau\right)}{\Delta_{l, r}(\tau)}
$$

where " $l$ " and " $r$ " refers to the left and right discrete states respectively, by assuming that locally $p=\rho R^{L}$ behaves as $K_{l, r} \tau^{-3}$ with

$$
K_{l, r}=\frac{1}{2}\left(\tau_{l}^{2} R_{l}^{L}+\tau_{r}^{2} R_{r}^{L}\right) .
$$

Then, the local counterpart of Whitham's condition is given by the inequality:

$$
a_{0}^{2}>K_{l, r}\left(\tau_{l}^{-2}+\left(\tau_{l} \tau_{r}\right)^{-1}+\tau_{r}^{-2}\right)\left(\tau_{l} \tau_{r}\right)^{-1},
$$

and the associated lower bound for $a_{0}$ is denoted by: $a_{0}^{W}=K_{l, r}\left(\tau_{l}^{-2}+\left(\tau_{l} \tau_{r}\right)^{-1}+\tau_{r}^{-2}\right)\left(\tau_{l} \tau_{r}\right)^{-1}$. 
- If we assume a particular expression in Eqs.(4): $\rho R^{L}=\pi(\rho)$ with $\pi \in \mathcal{C}^{\infty}(] 0,+\infty[)$, such that $\pi^{\prime}(\rho)>0$ for all $\rho>0$, in order to prevent the relaxation system (7) (with $\Psi=a_{0}^{2} \vartheta$ ) from instabilities, we need to comply with Whitham's condition (see for instance $[4,5])$ :

$$
a_{0} \vartheta>c_{\phi} .
$$

where $c_{\phi}$ is the celerity corresponding to original system (4). In this particular case, we can calculate the speed of acoustic waves:

$$
c_{\phi}^{2}=\pi^{\prime}(\rho) .
$$

For the isentropic Euler equations with $\gamma=3$ (which besides corresponds to the approach A1), we have an explicit formula:

$$
\rho R=S_{0} \rho^{3} \quad \text { with } \quad S_{0}=\frac{R_{0}}{\rho_{0}^{2}} .
$$

Then,

$$
c_{\phi}=\sqrt{3 R_{0}}\left(\frac{\rho}{\rho_{0}}\right)
$$

and, taking $\rho=\max \left(\rho_{l}, \rho_{r}\right)$, Whitham's condition can be formulated as follows:

$$
a_{0}>\sqrt{3 R_{0}}\left(\frac{\rho^{2}}{\rho_{0}}\right)
$$

with a lower bound $a_{0}^{W}=\sqrt{3 R_{0}} \rho^{2} / \rho_{0}$.

Finally, we take $a_{0}=\max \left(a_{0}^{\lambda}, a_{0}^{W}\right)$.

\section{Appendix B. Energy control in the relaxation systems (A1/A2)}

In order to give an estimate of the mean kinetic energy, which is caracterising the initial system of equations (4), we study the evolution of the "total" energy in the relaxation system. We focus on smooth solutions in this appendix.

Let us denote by

$$
E(x, t)=\frac{1}{2}\left(\rho U^{2}\right)(x, t) \geq 0 \quad \text { and } \quad \mathbb{E}(t)=\int_{\Omega} E(x, t) d x \geq 0
$$

the kinetic energy of the drift (the mean motion), by

$$
K(x, t)=\frac{1}{2}(\rho R)(x, t) \geq 0 \quad \text { and } \quad \mathbb{K}(t)=\int_{\Omega} K(x, t) d x \geq 0
$$

the energy of fluctuating particle motion, and by

$$
\begin{gathered}
\mathcal{E}(x, t)=E(x, t)+K(x, t)=\frac{1}{2}\left(\rho U^{2}\right)(x, t)+\frac{1}{2}(\rho R)(x, t) \geq 0, \\
\Sigma(t)=\int_{\Omega} \mathcal{E}(x, t) d x=\mathbb{E}(t)+\mathbb{K}(t) \geq 0
\end{gathered}
$$

the "total" particle energy.

Approach (A1). We study the homogeneous relaxation system corresponding to the approach (A1): 


$$
\left\{\begin{array}{l}
\frac{\partial \rho}{\partial t}+\frac{\partial}{\partial x}(\rho U)=0 \\
\frac{\partial}{\partial t}(\rho U)+\frac{\partial}{\partial x}\left(\rho U^{2}\right)+\frac{\partial}{\partial x}(\rho R)=0 \\
\frac{\partial}{\partial t}(\rho R)+\frac{\partial}{\partial x}(\rho U R)+2 \rho R \frac{\partial U}{\partial x}=0
\end{array}\right.
$$

on the closed domain $\Omega \times[0, T]$ with realizable initial conditions and homogeneous Dirichlet boundary conditions on $U:\left.U(x, t)\right|_{\partial \Omega}=0$.

From $(75.1),(75.2)$ we obtain an equation for the mean energy evolution:

$$
\frac{\partial}{\partial t}\left(\frac{1}{2} \rho U^{2}\right)+\frac{\partial}{\partial x}\left(U \cdot \frac{1}{2} \rho U^{2}\right)+U \frac{\partial}{\partial x}(\rho R)=0
$$

or

$$
\frac{\partial E}{\partial t}+\frac{\partial}{\partial x}(U E)+\frac{\partial}{\partial x}(2 K U)-2 K \frac{\partial U}{\partial x}=0
$$

In the same manner, from (75.3) we develop an equation for the evolution of $K(x, t)$ :

$$
\frac{\partial K}{\partial t}+\frac{\partial}{\partial x}(U K)+2 K \frac{\partial U}{\partial x}=0
$$

Finally, we get an equation for the "total" energy variation:

$$
\frac{\partial \mathcal{E}}{\partial t}+\frac{\partial}{\partial x}(U \mathcal{E})+\frac{\partial}{\partial x}(2 U K)=0
$$

From this equation, owing to the homogeneous Dirichlet boundary conditions on $U$ it follows that

$$
\frac{\partial}{\partial t} \int_{\Omega} \mathcal{E} d x=0
$$

Thus, during the evolution step the total energy is conserved:

$$
\Sigma\left(t^{n+1,-}\right)=\Sigma\left(t^{n}\right)
$$

that can be rewritten in the form:

$$
\mathbb{E}\left(t^{n+1,-}\right)+\mathbb{K}\left(t^{n+1,-}\right)=\mathbb{E}\left(t^{n}\right)+\mathbb{K}\left(t^{n}\right) .
$$

If we assume that:

- $K\left(x, t^{n}\right) \geq 0$ is ensured by initial conditions,

- $\forall t \in\left[t^{n}, t^{n+1}\right], K\left(x_{\Gamma^{-}}, t^{n}\right) \geq 0$ on $\Gamma^{-}=\{x \in \partial \Omega \mid U(x, t) . n<0\}$, where $n$ denotes the outward normal,

- $\forall t \in\left[t^{n}, t^{n+1}\right], U(x, t)$ and $\frac{\partial U}{\partial x}(x, t) \in L^{\infty}\left(\Omega \times\left[t^{n}, t^{n+1}\right]\right)$,

then on $\Omega \times\left[t^{n}, t^{n+1,-}\right] K(x, t)$ solution of (78) remains positive. Thus:

$$
\mathbb{K}\left(t^{n+1,-}\right)=\int_{\Omega} K\left(x, t^{n+1,-}\right) d x \geq 0,
$$

where $K\left(x, t^{n+1,-}\right)$ is solution of (78).

We have also:

$$
\mathbb{E}\left(t^{n+1}\right)=\mathbb{E}\left(t^{n+1,-}\right)
$$


since $E=E(\rho, U)$, and $\rho^{n+1}=\rho^{n+1,-}, U^{n+1}=U^{n+1,-}$.

Finally, since $\left(\mathbb{E}\left(t^{n}\right)+\mathbb{K}\left(t^{n}\right)\right)$ is bounded we obtain from (82) the following estimate of the energy $\mathbb{E}\left(t^{n+1}\right)$ :

$$
0 \leq \mathbb{E}\left(t^{n+1}\right)=\mathbb{E}\left(t^{n}\right)+\mathbb{K}\left(t^{n}\right)-\mathbb{K}\left(t^{n+1,-}\right) \leq \mathbb{E}\left(t^{n}\right)+\mathbb{K}\left(t^{n}\right) .
$$

Thus $\mathbb{E}\left(t^{n+1}\right)=\mathbb{E}\left(\rho^{n+1}, U^{n+1}\right)$ is bounded.

Approach (A2). Now, we apply the same reasoning to obtain the estimate for the mean kinetic energy for the relaxation system corresponding to approach (A2):

$$
\left\{\begin{array}{l}
\frac{\partial \rho}{\partial t}+\frac{\partial}{\partial x}(\rho U)=0 \\
\frac{\partial}{\partial t}(\rho U)+\frac{\partial}{\partial x}\left(\rho U^{2}+\rho R\right)=0 \\
\frac{\partial}{\partial t}(\rho R)+\frac{\partial}{\partial x}(\rho U R)+\left(a_{0}^{2} \vartheta-\rho R\right) \frac{\partial U}{\partial x}=0
\end{array}\right.
$$

Keeping the notations (71) - (74), we have the same equation for the mean kinetic energy evolution:

$$
\frac{\partial E}{\partial t}+\frac{\partial}{\partial x}(U E)+\frac{\partial}{\partial x}(2 K U)-2 K \frac{\partial U}{\partial x}=0
$$

The counterpart of (78) for the evolution of the fluctuating motion energy $K(x, t)$ is:

$$
\frac{\partial K}{\partial t}+\frac{\partial}{\partial x}(U K)+2 K \frac{\partial U}{\partial x}+\frac{1}{2}\left(a_{0}^{2} \vartheta-3 \rho R\right) \frac{\partial U}{\partial x}=0
$$

But here, we can not obtain a conservative equation for the "total" energy evolution. Actually, the sum of the last two equations results in a non-conservative equation:

$$
\frac{\partial \mathcal{E}}{\partial t}+\frac{\partial}{\partial x}(U \mathcal{E})+\frac{\partial}{\partial x}(2 U K)+\frac{1}{2}\left(a_{0}^{2} \vartheta-3 \rho R\right) \frac{\partial U}{\partial x}=0 .
$$

For this reason, we introduce a slightly modified definition of the total energy, which is now called $\mathcal{E}^{\prime}$ :

$$
\mathcal{E}^{\prime}=\mathcal{E}+\rho g(\omega)=E+K+\rho g(\omega)
$$

where

$$
\omega(\rho, R)=a_{0}^{2} \vartheta-3 \rho R
$$

We note that $\omega$ may be viewed as a "small parameter" that measures the drift between (A1) and (A2).

For any solution $(\rho, R)$ of the system $(86)$, the function $g(\omega)$ complies with:

$$
\frac{\partial(\rho g)}{\partial t}+\frac{\partial}{\partial x}(\rho U g)-4 a_{0}^{2} g^{\prime}(\omega) \frac{\partial U}{\partial x}=0
$$

Moreover, we have:

$$
\frac{\partial \mathcal{E}}{\partial t}+\frac{\partial}{\partial x}(U \mathcal{E})+\frac{\partial}{\partial x}(2 U K)+\frac{\omega}{2} \frac{\partial U}{\partial x}=0
$$

The sum of these two gives us the equation for the "total" energy $\mathcal{E}^{\prime}$ evolution:

$$
\frac{\partial}{\partial t}(\mathcal{E}+\rho g)+\frac{\partial}{\partial x}(U(\mathcal{E}+\rho g))+\frac{\partial}{\partial x}(2 U K)+\left(\frac{\omega}{2}-4 a_{0}^{2} g^{\prime}(\omega)\right) \frac{\partial U}{\partial x}=0 .
$$

If we choose

$$
g(\omega)=\frac{\omega^{2}}{16 a_{0}^{2}} \geq 0
$$


we can rewrite equations for $E, K$ and $(\rho g)$ as follows:

$$
\left\{\begin{array}{l}
\frac{\partial E}{\partial t}+\frac{\partial}{\partial x}(U E)+\frac{\partial}{\partial x}(2 K U)-2 K \frac{\partial U}{\partial x}=0 \\
\frac{\partial K}{\partial t}+\frac{\partial}{\partial x}(U K)+2 K \frac{\partial U}{\partial x}+\frac{\omega}{2} \frac{\partial U}{\partial x}=0 \\
\frac{\partial(\rho g)}{\partial t}+\frac{\partial}{\partial x}(U \rho g)-\frac{\omega}{2} \frac{\partial U}{\partial x}=0
\end{array}\right.
$$

Hence, equation (94) for the energy $\mathcal{E}^{\prime}$ evolution is conservative:

$$
\frac{\partial \mathcal{E}^{\prime}}{\partial t}+\frac{\partial}{\partial x}\left(U \mathcal{E}^{\prime}\right)+\frac{\partial}{\partial x}(2 U K)=0 .
$$

Thus, the total energy $\mathcal{E}^{\prime}$ is conserved during the evolution step:

$$
\Sigma^{\prime}(t)=\int_{\Omega} \mathcal{E}^{\prime}(x, t) d x, \quad \Sigma^{\prime}\left(t^{n+1,-}\right)=\Sigma^{\prime}\left(t^{n}\right),
$$

still using homogeneous Dirichlet boundary conditions for $U(x, t)$. This is equivalent to:

$$
\mathbb{E}\left(t^{n+1,-}\right)+\mathbb{K}\left(t^{n+1,-}\right)+\int_{\Omega}(\rho g)\left(x, t^{n+1,-}\right) d x=\mathbb{E}\left(t^{n}\right)+\mathbb{K}\left(t^{n}\right)+\int_{\Omega}(\rho g)\left(x, t^{n}\right) d x,
$$

or:

$0 \leq \mathbb{E}\left(t^{n+1}\right)=\mathbb{E}\left(t^{n+1,-}\right)=\mathbb{E}\left(t^{n}\right)+\mathbb{K}\left(t^{n}\right)+\int_{\Omega}(\rho g)\left(x, t^{n}\right) d x-\mathbb{K}\left(t^{n+1,-}\right)-\int_{\Omega}(\rho g)\left(x, t^{n+1,-}\right) d x$.

Hence:

$$
0 \leq \mathbb{E}\left(t^{n+1}\right) \leq \mathbb{E}\left(t^{n}\right)+\mathbb{K}\left(t^{n}\right)+\int_{\Omega}(\rho g)\left(x, t^{n}\right) d x-\mathbb{K}\left(t^{n+1,-}\right)
$$

since $(\rho g)(x, t) \geq 0$.

\section{Appendix C. On hybrid modelling of dispersed turbulent two-phase flows}

Poly-dispersed two-phase flows consisting of a turbulent carrier phase (a liquid or a gas) and dispersed particles (solid particles, droplets or bubbles) are complex processes which are very important in many industrial situations. Therefore, an accurate prediction of these flows is required for engineering purposes. Furthermore, for the modelling and numerical simulation of a poly-dispersed two-phase flow, the two phases (fluid and particles phase) have to be treated in a coupled way.

However, the two most popular approaches (Eulerian and Lagrangian) for two-phase flow modelling have disadvantages which limit their capacities. Hybrid methods try to gather the advantages of the Eulerian approach (expected values free from statistical error and low calculation costs) with those of the Lagrangian approach (polydispersity and non-linear local source terms are treated without approximations) [31, 32]. At the moment, most hybrid methods use only one description (Eulerian or Lagrangian) for each phase. The idea of the hybrid method introduced in [10] is to use a mixed Eulerian/Lagrangian formulation for the dispersed phase while the properties of fluid remain calculated with the Eulerian approach. By treating some statistical quantities (such as the mean particle velocity and the local particle concentration) in an Eulerian manner (i.e. as mean fields defined on a fixed grid), the overall system is less sensitive to errors introduced by statistical noise. This, in turn, will eventually allow for computations using a smaller number of computational particles, thus leading to faster calculations. 
Therefore, in this new hybrid method the two approaches are coupled in a fully consistent manner while keeping the high level of physical information provided by the Lagrangian description. The incompressible fluid phase is described by Reynolds Averaged Navier-Stokes (RANS) equations which are solved by an Eulerian Finite Volume solver (that is not detailed here). Then, the fluid-related mean fields (i.e. mean pressure $\langle P\rangle$ (or its gradient), mean fluid velocity $\left\langle\mathbf{U}_{f}\right\rangle$, Reynolds stress tensor $\mathbf{R}=\left\{R_{i j}\right\}_{i, j=1}^{d}$, with $d$-space dimensions and the dissipation rate of the turbulent kinetic energy $\langle\epsilon\rangle$ ) are provided to the particle-phase model. The flows we are dealing with are considered to be dilute enough in particles not to consider the turbulence modulation. The dispersed (particle) phase is modelled by a coupled Eulerian/Lagrangian description. The Lagrangian part of the model is given by stochastic differential equations (SDEs), which are solved by resorting to particle/mesh Monte-Carlo methods [34]. The Eulerian part of the particle model is given by a system of partial differential equations for mean quantities which present an important convective part. These equations describe the time evolution of the first order moments of the particle volume fraction and the instantaneous particle velocity and are deduced from Lagrangian stochastic description ([31], chap.6.7). Thus, both systems are consistent by construction.

In order to guarantee the coupling of methods for the particle description we have to choose the averaging and interpolation procedures, that is how information is exchanged from the mesh to the stochastic particles (interpolation of Eulerian mean fields at particle positions) and from the stochastic particles to the mesh (how mean fields are extracted). Moreover, such a coupling introduces noisy quantities (computed by the stochastic equations) in the Eulerian part of the model.

\section{An example of the particle phase simulation using hybrid method}

In this appendix we present a two-dimensional case of turbulent two-phase wall jet, where solid particles are involved in the turbulent air flow, which is characterized by the non-homogeneous anisotropic stationary turbulence. Only the particle hybrid (Eulerian/Lagrangian) model is solved, whereas the fluid mean fields are provided by a preliminary single-phase computation and are taken as frozen fields. We consider heavy enough particles in a such a way that the gravity and the drag forces are predominant in a two-dimensional framework, and we intend to compare the first order particle moments computed using the present hybrid model (these will be referred to as Eulerian coupled approximations) with those provided by a stand alone Lagrangian computation ("pure" Lagrangian) for the particle phase in the same flow configurations.

\section{Mean-field/PDF hybrid model and experimental setup}

We consider a rectangular domain of dimensions $L x \times L y$. A sketch of the geometry is given in Fig.16, where $\mathrm{AE}$ is a wall boundary. Air at ambient temperature and atmospheric pressure is injected both in $\mathrm{AB}$ and $\mathrm{BC}$ regions. Solid particles are injected in $\mathrm{AB}$ region of diameter $d$ and after some time leave the domain by outlets $\mathrm{CD}$ and DE. A stationary turbulent two-phase flow is eventually obtained.

- The Lagrangian stochastic description of the dispersed phase is given by the Simple Langevin Model (SLM) [31]:

$$
\left\{\begin{array}{l}
d x_{p, i}=U_{p, i} d t, \\
d U_{p, i}=\frac{U_{s, i}-U_{p, i}}{\tau_{p}} d t, \quad i=1,2, \quad t>0, \\
d U_{s, i}=-\frac{1}{\rho_{f}} \frac{\partial\langle P\rangle}{\partial x_{i}} d t-\frac{U_{s, i}-\left\langle U_{f, i}\right\rangle}{T_{L}} d t+\sqrt{C_{0}\langle\varepsilon\rangle} d W_{t, i},
\end{array}\right.
$$

where $\mathbf{x}_{p}(t)$ the particle position, $\mathbf{U}_{p}(t)$ the particle velocity, $\mathbf{U}_{s}(t)$ the fluid velocity seen at the particle position and $\mathbf{W}(t)$ the vector of independent Wiener processes, all variables 


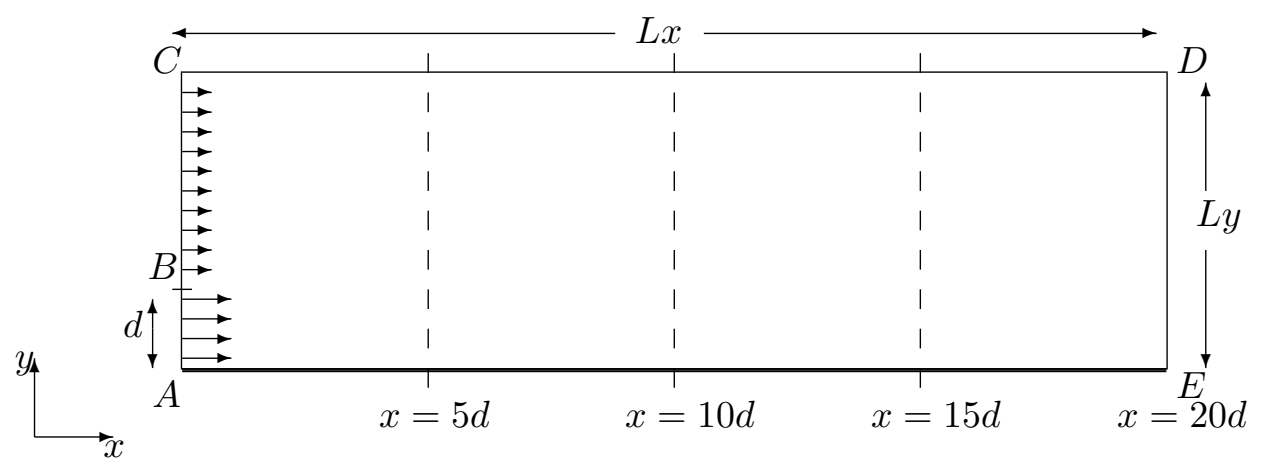

Figure 16: Computational domain, definition of the geometry of a wall jet of diameter $d$.

(random processes) being defined on the same probability space [33]. The particle relaxation time scale $\tau_{p}$ is a function of the particle inertia and represents the time necessary for a particle to adjust to fluid velocities, $T_{L}$ stands for the fluid integral timescale. The fluid mean fields $k,\langle\varepsilon\rangle,\left\langle\mathbf{U}_{f}\right\rangle,\langle P\rangle$ are external data computed in advance with $k-\varepsilon$ model and then used as a frozen field. Following Monte-Carlo algorithm, particle related statistics can be obtained from equations (102) for a given averaging operator. In practice, at each cell $j \in \mathbb{Z}$ of the mesh (with the cell center $\mathbf{x}_{\mathbf{j}}$ ) containing $N_{p}^{[j]}$ particles at the time $t$, the following approximation is used to compute the particle volumetric fraction $\alpha_{p}$ :

$$
\alpha_{p}\left(t, \mathbf{x}_{\mathbf{j}}\right)=\frac{\sum_{n=1}^{N_{p}^{[j]}} v^{(n)}}{\mathcal{V}_{j}},
$$

where $v^{(n)}$ is the volume occupied by a particle and $\mathcal{V}_{j}$ - the volume of the cell $j, j \in \mathbb{Z}$. For other mean particle quantities, we obtain the numerical approximations as mean averages:

$$
\left\langle H_{p}\right\rangle\left(t, \mathbf{x}_{\mathbf{j}}\right) \simeq\left\langle H_{p}\right\rangle_{N_{p}}\left(t, \mathbf{x}_{\mathbf{j}}\right)=\frac{\sum_{n=1}^{N_{p}^{[j]}} H_{p}^{(n)}(t)}{N_{p}^{[j]}} .
$$

- The Eulerian part of the hybrid model describes the time evolution of the first order statistical moments: the mean particle density, $\rho_{p}=\alpha_{p} \rho_{p}^{0}$ with constant $\rho_{0}$, and the instantaneous mean particle velocity, $\left\langle\mathbf{U}_{p}\right\rangle$, whose governing equations are

$$
\left\{\begin{array}{l}
\partial_{t} \rho_{p}+\partial_{x_{j}}\left(\rho_{p}\left\langle U_{p, j}\right\rangle\right)=0, \quad t>0, \quad \mathbf{x} \in \mathbb{R}^{2}, \\
\partial_{t}\left(\rho_{p}\left\langle U_{p, i}\right\rangle\right)+\partial_{x_{j}}\left(\rho_{p}\left\langle U_{p, i}\right\rangle\left\langle U_{p, j}\right\rangle\right)+\partial_{x_{j}}\left(\rho_{p} R_{p, i j}^{L}\right)=\rho_{p} g_{i}+\rho_{p}\left\langle U_{r, i}^{L} / \tau_{p}^{U}\right\rangle,
\end{array}\right.
$$

where $\tau_{p}^{U}=\tau_{p}^{L}$ is the particle velocity relaxation time scale and $\mathbf{U}_{r}^{L}=\left(\mathbf{U}_{s}-\mathbf{U}_{p}\right)^{L}$ is the local relative velocity. The subscript " $p$ " is used to emphasize the particle related variables, whereas the superscript " $L$ " now refers to variables computed using Lagrangian description (102). For instance, the particle related kinetic tensor $R_{p, i j}=\left\langle u_{i}^{\prime} u_{j}^{\prime}\right\rangle(i, j=$ $1,2)$ is computed using (104) and provided to the Eulerian particle model (105). In both Eulerian and Lagrangian descriptions, Reynolds decomposition takes place, thus $\mathbf{u}_{p}^{\prime}(t, \mathbf{x})=$ $\mathbf{U}_{p}(t, \mathbf{x})-\left\langle\mathbf{U}_{p}(t, \mathbf{x})\right\rangle$. Right-hand side terms in the second equation of (105) describe the gravity and the drag forces. It is important to note that equations (105) have been actually deduced from system (102) ([31], chap.6.7). Thus, both Eulerian and Lagrangian descriptions are consistent by construction.

\section{A two-dimensional relaxation scheme}

As it was emphasized above, the coupling of descriptions (102), (105) introduces non-smooth quantities in the Eulerian part of the model (105), that can result in instabilities during numerical simulations. The present section aims at introducing a two-dimensional relaxation scheme 
for simulation of system (105) that could enable stable approximations in a number of limit situations. From now on, we omit the subscripts " $p$ " and denote by $\rho$ the mean particle density and by $U_{i}=\left\langle U_{p, i}\right\rangle, i=1,2$ the mean particle velocity. Hence, for given non-smooth values of the Lagrangian kinetic tensor $R_{i j}^{L}=\left\langle u_{p, i}^{\prime} u_{p, j}^{\prime}\right\rangle^{L}, \forall i, j \in \mathbb{Z}$, we want to compute stable approximations of solutions of:

$$
\left\{\begin{array}{l}
\partial_{t} \rho+\partial_{x_{j}}\left(\rho U_{j}\right)=0, \quad t>0, \quad \mathbf{x} \in \mathbb{R}^{2} \\
\partial_{t}\left(\rho U_{i}\right)+\partial_{x_{j}}\left(\rho U_{i} U_{j}\right)+\partial_{x_{j}}\left(\rho R_{i j}^{L}\right)=\rho g_{i}+\rho\left\langle U_{r, i} / \tau_{p}\right\rangle^{L} .
\end{array}\right.
$$

By construction, $R_{i j}^{L}, i, j=1,2$ complies with the realisability condition:

$$
x_{i} R_{i j}^{L} x_{j} \geq 0 \quad \forall x_{i}, x_{j}(i, j=1,2) .
$$

On the basis of $[3,27]$, getting rid of physical source terms $\left(\rho g_{i}+\rho\left\langle U_{r, i} / \tau_{p}\right\rangle^{L}\right), i=1,2$, the following relaxation system naturally arises:

$$
\left\{\begin{array}{l}
\partial_{t} \rho+\partial_{x_{j}}\left(\rho U_{j}\right)=0, \quad t>0, \quad \mathbf{x} \in \mathbb{R}^{2} \\
\partial_{t}\left(\rho U_{i}\right)+\partial_{x_{j}}\left(\rho U_{i} U_{j}\right)+\partial_{x_{j}}\left(\rho R_{i j}\right)=0 \\
\partial_{t}\left(\rho R_{i j}\right)+\partial_{x_{k}}\left(\rho U_{k} R_{i j}\right)+\rho\left(R_{i k} \partial_{x_{k}} U_{j}+R_{j k} \partial_{x_{k}} U_{i}\right)=\rho\left(R_{i j}^{L}-R_{i j}\right) / \tau_{p}^{R} .
\end{array}\right.
$$

This system is invariant under frame rotation. If we consider the reference frame $(\mathbf{n}, \tau)$, where $\mathbf{n}=\left(n_{x}, n_{y}\right), \tau=\left(-n_{y}, n_{x}\right)$, such that $n_{x}^{2}+n_{y}^{2}=1$, we may introduce: $U_{n}=\mathbf{U} . \mathbf{n}, U_{\tau}=\mathbf{U} \cdot \tau$, $R_{n n}=\mathbf{n}^{t} . \mathbf{R} . \mathbf{n}, R_{n \tau}=\mathbf{n}^{t} . \mathbf{R} . \tau=\tau^{t} \mathbf{R} . \mathbf{n}=R_{\tau n}, R_{\tau \tau}=\tau^{t} . \mathbf{R} . \tau$. While neglecting transverse variations, the homogeneous system corresponding to (108) written in terms of the new variable $Z_{2 D}=\left(\rho, U_{n}, U_{\tau}, \rho R_{n n}, \rho R_{n \tau}, S\right)^{t}$ with $S=\left(\left(\rho R_{n n}\right)\left(\rho R_{\tau \tau}\right)-\left(\rho R_{n \tau}\right)^{2}\right) / \rho^{4}$ takes the following form for smooth solutions:

$$
\partial_{t} Z_{2 D}+A_{n}\left(Z_{2 D}\right) \partial_{n} Z_{2 D}=0
$$

with system marix

$$
\begin{gathered}
A_{n}\left(Z_{2 D}\right)=\left(\begin{array}{cccccc}
U_{n} & \rho & 0 & 0 & 0 & 0 \\
0 & U_{n} & 0 & \vartheta & 0 & 0 \\
0 & 0 & U_{n} & 0 & \vartheta & 0 \\
0 & \Psi_{n n} & 0 & U_{n} & 0 & 0 \\
0 & 2 \rho R_{n \tau} & \Phi_{n \tau} & 0 & U_{n} & 0 \\
0 & 0 & 0 & 0 & 0 & U_{n}
\end{array}\right), \\
\text { where: } \Psi_{n n}=3 \rho R_{n n}, \quad \Phi_{n \tau}=\rho R_{n n},
\end{gathered}
$$

and $Z_{2 D}=Z_{2 D}\left(t, x_{n}\right)$. This method associated with the choice (110) is approach (A1) introduced in [27] and defined in the one-dimensional framework in sec. 2.2 of the present paper.

Then, by denoting $\vartheta(x, t)=1 / \rho(x, t)$ and choosing constant $\left(R_{n n}\right)_{0} \in \mathbb{R}_{+}^{*}$, we may introduce in a two-dimensional framework approach (A2) as corresponding to the choice:

$$
\Psi_{n n}=3 \rho_{0}^{2}\left(R_{n n}\right)_{0} \vartheta, \quad \Phi_{n \tau}=\rho_{0}^{2}\left(R_{n n}\right)_{0} \vartheta .
$$

We note that the relaxation system corresponding to system (109) together with choice (111) is hyperbolic and only admits LD fields. A more detailed presentation can be found in $[15,16]$ and [18].

\section{Numerical simulation}

In present computations we use the following dimensions: $L x=1.0 \mathrm{~m}, L y=0.2 \mathrm{~m}, \mathrm{~d}=$ $0.05 \mathrm{~m}$. The fluid is characterized by the density $\rho_{f}=2.1 \mathrm{~kg} / \mathrm{m}^{3}$, the dynamical viscosity $\mu_{f}=1.85 \times 10^{-5}$ Pa.s and $C_{0}=2.1$. The normal components of the inlet gas velocity are: 
$1.5 \mathrm{~m} / \mathrm{s}$ in region $\mathrm{AB}$ and $1.0 \mathrm{~m} / \mathrm{s}$ in $\mathrm{BC}$. The dispersed phase is described by the particle density $\rho_{p}=2000 \mathrm{~kg} / \mathrm{m}^{3}$ and the diameter $d_{p}=10^{-5} \mathrm{~m}$. The computations are performed on a uniform mesh with $N x \times N y=200 \times 40$ cells and $\Delta t=10^{-3} \mathrm{~s}$. At the beginning of each time step, particles are injected in the domain with the fluid velocity. For a particle $(n)$ injected in the cell $[i]$ of the domain, we assume the following initial conditions:

$$
\mathbf{U}_{p}^{(n)}=\left\langle\mathbf{U}_{f}\right\rangle^{[i]}, \quad \mathbf{U}_{s}^{(n)}=\left\langle\mathbf{U}_{f}\right\rangle^{[i]}+\sqrt{\frac{2}{3} k^{[i]}} \zeta,
$$

where $\zeta=\left(\zeta_{x}, \zeta_{y}\right)$ is a vector of independent $\mathcal{N}(0,1)$ random variables. For such a configuration, $\tau_{p}$ is equal approximately $6 \times 10^{-4} \mathrm{~s}$. When the stationary state is reached, the total number of particles present in the domain at each time $t$ varies around $N_{p}=3.2 \times 10^{5}$.

Finally, we are interested by the first order particle statistics only, more precisely, by the particle volume fraction $\alpha_{p}$ and the mean particle flow rate $\left(\alpha_{p} \rho_{0}\left\langle\mathbf{U}_{p}\right\rangle\right)$. We compare the results computed by the Eulerian part (105) of the hybrid model, where the kinetic tensor is provided by system (102), with those obtained with a "stand-alone" Lagrangian computation using "pure" PDF description (102). The profiles of the solution corresponding to the cross-stream sections $x=0 d, 5 d, 10 d, 15 d$ are shown in Fig. $17-19$.

Particle volume fraction with $200 \times 40$ cells, $\mathrm{Np}=8 \times 10^{4}, \mathrm{~T}=6.0 \mathrm{~s}$
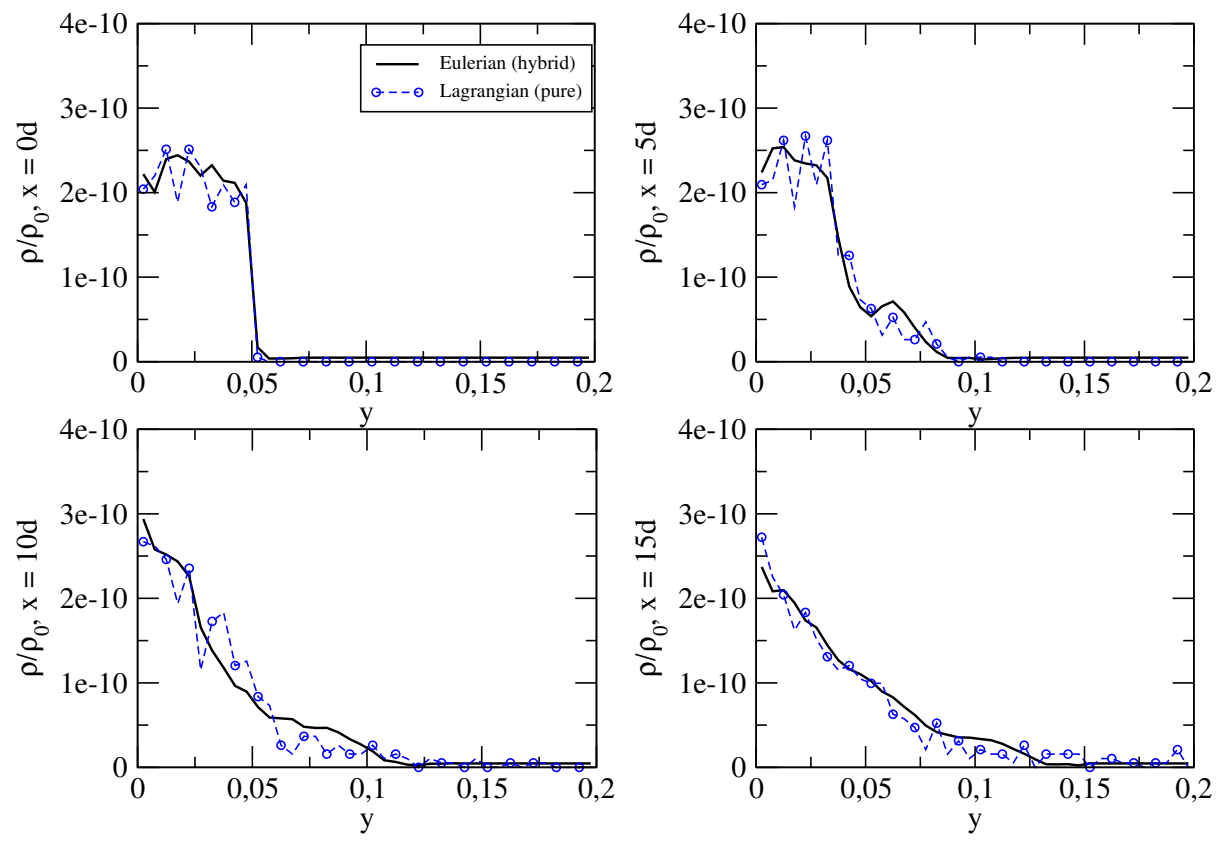

Figure 17: Particle volume fraction. Numerical simulation of hybrid model (102), (105) with $N_{p}=$ $8 \times 10^{4}$ particles on the mesh of $200 \times 40$ cells with $\Delta t=10^{-3} s$. Comparison with the results of the stand-alone Lagrangian computation. 
Particle flow rate, Qx, with $200 \times 40$ cells, $\mathrm{N}_{\mathrm{p}}=8 \times 10^{4}, \mathrm{~T}=6.0 \mathrm{~s}$
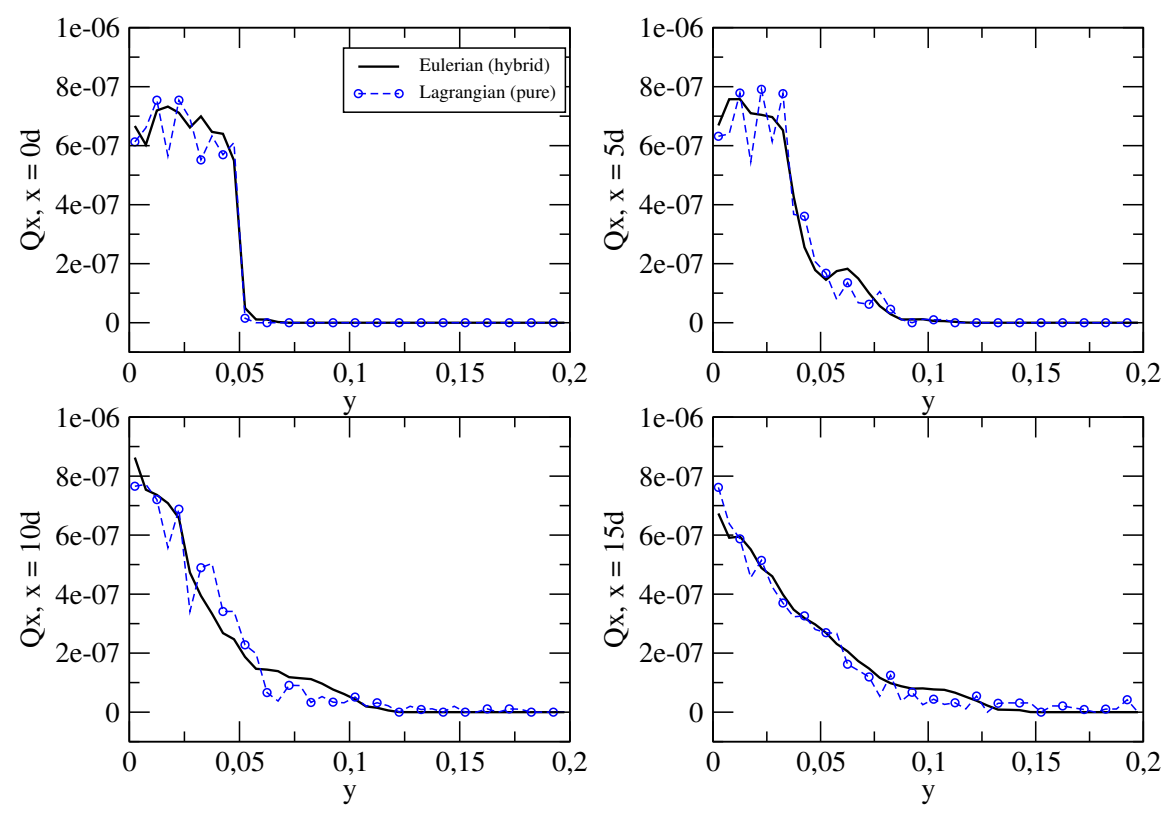

Figure 18: Mean particle flow rate in the longitudinal direction. Numerical simulation of hybrid model (102), (105) with $N_{p}=8 \times 10^{4}$ particles on the mesh of $200 \times 40$ cells with $\Delta t=10^{-3} s$. Comparison with the results of the stand-alone Lagrangian computation.

Particle flow rate, Qy, with $200 \times 40$ cells, $\mathrm{Np}=8 \times 10^{4}, \mathrm{~T}=6.0 \mathrm{~s}$
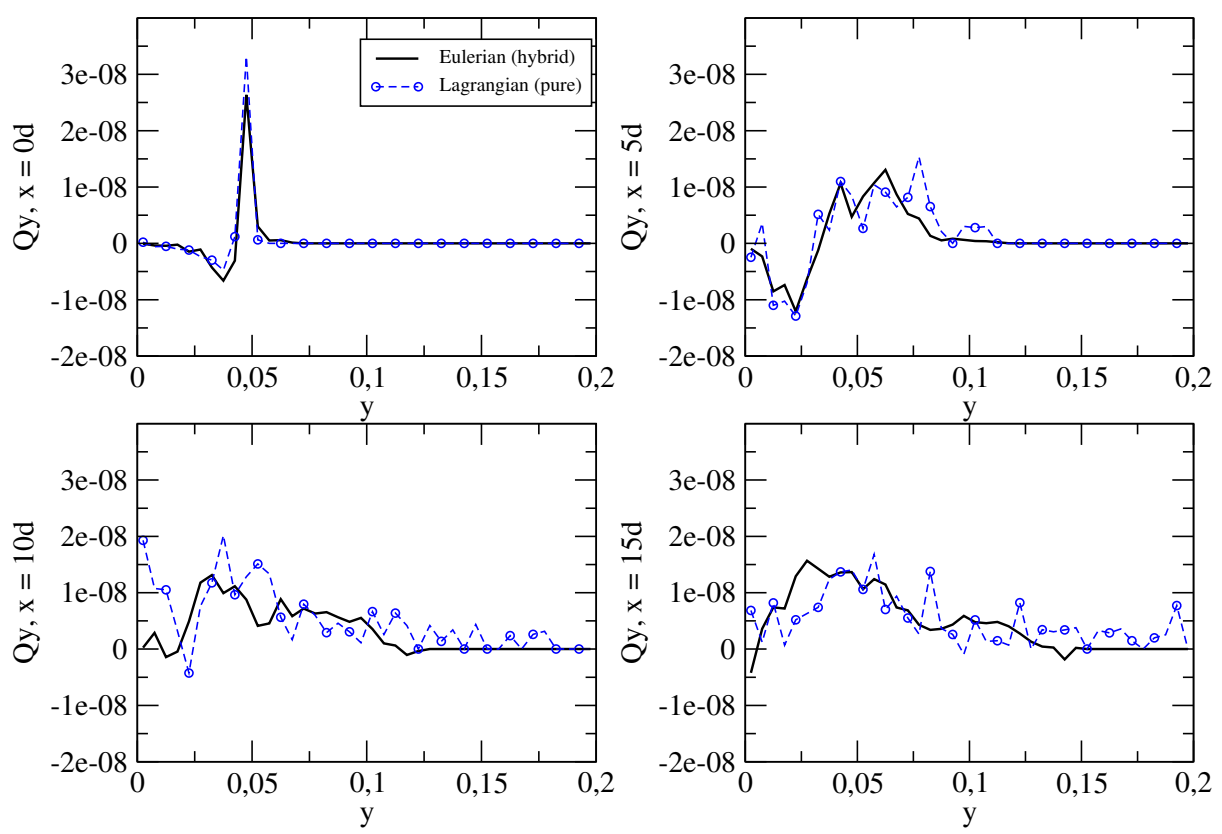

Figure 19: Mean particle flow rate in the transversal direction. Numerical simulation of hybrid model (102), (105) with $N_{p}=8 \times 10^{4}$ particles on the mesh of $200 \times 40$ cells with $\Delta t=10^{-3} s$. Comparison with the results of the stand-alone Lagrangian computation. 


\section{References}

[1] A. Ambroso, C. Chalons, F. Coquel and T. Galié, "Relaxation and numerical approximation of a two-fluid two-pressure diphasic model", ESAIM: M2AN, vol. 43 (6), pp. 1063-1097, 2009.

[2] B. Audebert, "Contribution à l'analyse de l'interaction onde de choc - couche limite", Université Pierre et Marie Curie, $\mathrm{PhD}$ thesis, 2006.

[3] C. Berthon, F. Coquel, J.M. Hérard and M. Uhlmann, "An approximate solution of the Riemann problem for a realisable second-moment turbulent closure", Shock Waves Journal, vol. 11 (4), pp. 245-269, 2002.

[4] F. Bouchut, "Nonlinear stability of finite volume methods for hyperbolic conservation laws and well-balanced schemes for source", Frontiers in Mathematics Series, Birkhauser, 2004.

[5] F. Bouchut, "A reduced stability condition for nonlinear relaxation to conservation laws", J. Hyp. Diff. Eq., vol 1(1), pp. 149-170, 2004.

[6] G. Brun, J.M. Hérard, D. Jeandel and M. Uhlmann, "An approximate Riemann solver for a class of realizable second order closures", Int. J. of Comp. Fluid Dynamics, vol. 13, n 3, pp. 223-249, 1999.

[7] T. Buffard, T. Gallouet and J.M. HÉrard, "Un schéma simple pour les équations de Saint-Venant", C. R. Acad. Sci. Paris, t. 326, Serie I, pp. 385-390, 1998.

[8] T. Buffard, T. Gallouet and J.M. Hérard, "A sequel to a rough Godunov scheme: application to real gases", Computers and Fluids, t. 29, pp. 813-847, 2000.

[9] C. Chalons, J.F. Coulombel, "Relaxation approximation of the Euler equations", $J$. Math. Anal. Appl., vol. 348, pp. 872-893, 2008.

[10] S. Chibbaro, J.M. Hérard and J.P. Minier, "A novel Hybrid Moments/MomentsPDF method for turbulent two-phase flows", Final Technical Report Activity Marie Curie Project. TOK project LANGE Contract MTKD-CT-2004 509849, 2006.

[11] L. Combe, "Simulation numérique d'écoulements gaz-particules sur maillage non structuré", Institut National Polytechnique de Toulouse, PhD thesis, 1997.

[12] F. Coquel, E. Godlewski, A. In, B. Perthame and P. Rascle, "Some new Godunov and relaxation methods for two phase flows", Proceedings of the International Conference on Godunov methods : theory and applications, Kluwer Academic, Plenum Publisher, 2001.

[13] F. Coquel, E. Godlewski, Seguin, N., "Relaxation of fluid systems", Mathematical Models and Methods in Applied Sciences, 2011.

[14] R. Courant, K. Friedrichs and H. Lewy, "On the partial difference equations of mathematical physics", IBM Journal, vol. 11, pp. 215-234, 1967.

[15] K. Dorogan, "Numerical schemes for the hybrid modelling of turbulent gas-particle flows", Université Aix-Marseille 1, $\mathrm{PhD}$ thesis to appear in 2012.

[16] K. Dorogan, J.M. HÉrard, " A two-dimensional relaxation scheme for hybrid modelling of gas-particle two-phase flows", EDF report, 2012, unpublished.

[17] K. Dorogan, J.M. Hérard, J.P. Minier, "Development of a new scheme for hybrid modelling of gas-particle two-phase flows", EDF report H-I81-2010-2352-EN, pp. 1-50, 2010, unpublished.

[18] K. Dorogan, J.M. HÉrard, J.P. Minier, "A two-dimensional relaxation scheme for the hybrid modelling of two-phase flows", International Conference Finite Volumes for Complex Applications 6, Prague, 6-10 juin 2011. Published in proceedings "Finite Volumes for Complex Applications. Problems and Perspectives “, pp. 351-359, Springer-Verlag (2011), available on http://www.springerlink.com/. 
[19] R. Eymard, T. Gallouet and R. Herbin, "Finite Volume Methods", Handbook of Numerical Analysis, vol. VII, pp. 713-1020, 2000.

[20] T. Gallouet, J.M. Hérard and N. Seguin, "On the use of symmetrizing variables for vacuum", Calcolo, vol. 40 (3), pp. 163-194, 2003.

[21] S. Gavrilyuk, H. Gouin, "Geometric evolution of the Reynolds stress tensor in three-dimensional turbulence", European J. Mechanics B/Fluids, http://hal.archivesouvertes.fr/hal-00371444/en/, submitted, 2010.

[22] E. Godlewski and P.A. Raviart, "Numerical approximation of hyperbolic systems of conservation laws", $A M S$, vol. 118, Springer, 1996.

[23] S.K. Godunov, "A difference method for numerical calculation of discontinuous equations of hydrodynamics", Sbornik, pp. 271-300, 1959.

[24] P. Helluy, J.M. Hérard, H. Mathis and S. Muller, "A simple parameter-free entropy correction for approximate Riemann solvers", CR Mécanique, vol. 338, pp. 493498, 2010.

[25] J.M. HÉRARD, "A relaxation tool to compute hybrid Euler-Lagrange compressible models", AIAA paper 2006-2872, 36th AIAA FD Conference, http://www.aiaa.org, 2006.

[26] J.M. Hérard, J.P. Minier and S. Chibbaro, "A Finite Volume scheme for hybrid turbulent two-phase flow models", AIAA paper 2007-4587, 18th AIAA CFD Conference, http://www.aiaa.org, 2007.

[27] J.M. Hérard, M. Uhlmann and D.E. Van der Velden, "Numerical techniques for solving hybrid Eulerian-Lagrangian models for particulate flows", EDF report H-I81-20093961-EN, pp. 1-50, 2009, unpublished.

[28] S. Jin AND Z. XIN, "The relaxation schemes for systems of conservation laws in arbitrary space dimensions", Comm. Pure Appl. Math., vol 48, pp. 235-276, 1995.

[29] P.D. Lax, "Hyperbolic systems of conservation laws", II, Comm. Pure Appl. Math., vol. 10, pp. 537-566, 1957.

[30] R. LeVeque, "Numerical methods for conservation laws", Lectures in Mathematics, ETH, Birkhauser, 1990.

[31] J.P. Minier, E. Peirano, "The pdf approach to polydispersed turbulent two-phase flows", Physics reports, vol. 352 (1-3), pp. 1-214, 2001.

[32] M. Muradoglu, P. Jenny, S.B. Pope and D.A. Caughey, "A consistent hybrid finite-volume/particle method for the pdf equations of turbulent reactive flows", Journal of Computational Physics, vol. 154, pp. 342-371, 1999.

[33] B. ØKsendal, "Stochastic differential equations. An introduction with applications", Springer, Berlin, 1995.

[34] E. Peirano, S. Chibbaro, J. Pozorski and J.P. Minier, "Mean-field/PDF numerical approach for polydispersed turbulent two-phase flows", Progress in Energy and Combustion Science, http://www.sciencedirect.com, vol. 32, pp. 315-371, 2006.

[35] K. Saleh, F. Coquel, N. Seguin, "Relaxation and numerical approximation for fluid flows in a nozzle", submitted to publication, also in EDF report H-I81-2011-01469-EN, 2011.

[36] I. Suliciu, "On the thermodynamics of fluids with relaxation and phase transitions. Fluids with relaxation", Internat. J. Engrg. Sci., vol. 36, pp. 921-947, 1998.

[37] J. Whitham, "Linear and nonlinear waves", Wiley, 1974.

[38] W.-A. YonG, "Singular perturbations of first-order hyperbolic systems with stiff source terms", J. Differential Equations, vol. 155(1), pp. 89-132, 1999. 
Reply 3 on detailed comments from the report on "A relaxation scheme for hybrid modeling of gas-particle flows " by Dorogan, Herard, Minier for a publication in "Computers and Fluids"

- Main comments. Most of remarks made by the reviewer have been taken into account and helped us to improve the paper on the whole. Yet, we would like to emphasize that the system we are studying in the context of hybrid modelling is system (4), where tensor $\mathbf{R}^{\mathrm{L}}(t, \mathbf{x})$ is, except for the $1 \mathrm{D}$ case, not spheric at all and its independent components are not functions of $(\rho, \rho \mathbf{U})$. Thus, the system of barotropic Euler equations is a particular "test system" for (4), where the correct convergence of approximations can be verified (to our knowledge, no detailed evaluation of the $L^{1}$ norm of errors is available in the "relaxation" litterature, even for barotropic Euler equations). It is also important to point out that there is no correspondence that could be made between the relaxation techniques for barotropic Euler equations and 'relaxation' system (A1), the latter being deduced from $R_{i j}$ model of turbulent flows. However, the relaxation system associated with system (4) and choice (A2) (that is in fact deduced from (A1) in order to eliminate genuinely non-linear fields, see introduction and constraint (C4) on p.3) does correspond in the one-dimensional case to the relaxation system introduced for the simulation of the barotropic Euler equations, when $\rho R^{L}=\pi(\rho)$. No further correspondence can be made in a two-dimensional framework. In order to avoid any kind of confusion in the one-dimensional case, some precisions and references have been added in remark 1 , sec. 2.2. In fact, the paper does not pretend to introduce a new relaxation technique for the simulation of barotropic Euler equations (for instance, in the title it is "A relaxation scheme for hybrid modelling of gas-particle flows" and not "A new relaxation scheme ..."). We recall that one of its main objectives consists in the comparison of approaches (A1) and (A2) in terms of stability and convergence towards the correct solutions, especially for shocks, when $R^{L}$ has a particular form. This has been emphasized in the text and in the conclusion. As underlined in the previous answer to the reviewer, it was not quite obvious that (A1) would provide convergent approximations. Moreover, the main purpose of the work is to build numerical techniques in order to compute stable approximate solutions of hybrid models. It seems that both (A1) and (A2) enable such computations.

- Main modifications in sections 1-4. For the seak of clarity, we have brought many modifications in section 2 that presents a relaxation technique for the simulation of the hybrid model (4). For instance, new properties 1, 2, 3 correspond to former propositions 1, 2, 3 respectively, where property 3 , as underlined on p. 6 , is associated with Theorem 3 in [9]. Though we have kept some 'well known' results (concerning, for example, the solution of the associated Riemann problem and the properties of the relaxation system), in order to allow a fluent reading of the paper submitted for publication in Computers and Fluids, we have nonetheless added references to papers dealing with relaxation techniques, where these developments have been achieved before, for instance $[4,9]$. Section 3 is very short and only provides the basics of the algorithm for approach (A2), which is compulsory for those who want to use the method. For verification cases present in section 4 , where $\rho R^{L}=\pi(\rho)$, approach (A2) coincides with "classical" relaxation techniques for barotropic Euler equations as this has been underlined in the text (in Remark 1, p. 5 and in the beginning of sec. 4 ).

- Main modifications in appendices. Former appendix B on $a_{0}$ parameter evaluation is referred to as Appendix A in the current version and contains some estimates for a lower bound of $a_{0}$ in the general case $\rho R^{L} \neq \pi(\rho)$ that underline the difficulty, with additional reference to [9]. Former appendix $\mathrm{C}$ (now, B) on energy control in relaxation systems has changed a little in order to take into account remarks of the reviewer. In fact, it contains 
some continuous energy estimations, unlike in $[4,9]$, where emphasis is put on discrete approximations. Former appendix D containing the proof of the positivity of the cell density has been withdrawn since the proof is indeed classical. The sample result with noisy data present in former appendix $\mathrm{E}$ has been moved at the end of section 4, that now contains two parts: 4.1. Algorithm verification and 4.2. Numerical results for (A2) with noisy kinetic tensor.

- Extension to the 2D framework. Concerning a two-dimensional extension of the method, we have now merged former appendices $\mathrm{A}$ and $\mathrm{F}$ in appendix $\mathrm{C}$ and have reformulated some points in order to clarify the presentation of hybrid methods, following the proposition made by the reviewer. Moreover, we have added in appendix $\mathbf{C}$ the twodimensional relaxation systems corresponding to approaches (A1) and (A2), where (A1) is coming from $R_{i j}$ model as before and (A2) is again inspired from (A1). However, it does not seem possible to add the details of $2 \mathrm{D}$ algorithm in the appendix, a whole paper would be needed for this. For instance, report [16] in english will be included in $\mathrm{PhD}$ thesis of K. Dorogan [15] to appear in 2012. We note also that the proceedings [18] are already available on http://www.springerlink.com/.

1. Some minor remarks. In appendix $C$, the reviewer is still non convinced with the assumption of (homogeneous) Dirichlet boundary condition in an hyperbolic setting, what is $\Gamma^{-}$in this $1 D$ case, moreover if the value of $U(x, t)$ on the boundary $\partial \Omega$ has been assumed to satisfy homogeneous Dirichlet conditions. The energy control assumes that the solutions are smooth, equation (77) is obtained from the non conservative equations for smooth solutions, what for $W_{\Delta}$ ? Is there some dissipation principle?

In fact, appendix B (former appendix C) contains some continuous energy estimations, unlike in $[4,9]$, where emphasis is put on discrete approximations (for more details, see for instance Appendix A, part II). To our knowledge, no dissipation principle is available for system (4). The homogeneous Dirichlet boundary conditions are imposed in order to ensure that $U .\left.n\right|_{\partial \Omega}=0$. In this case, $\Gamma^{-}$is a null set.

2. Section 1. In the plan, the authors write about the energy control 'whatever $R^{L}$ ' is: indeed the energy control is studied for the homogenous part of a system where $R L$ is not involved.

This was in fact a mistake that has been corrected in the present version to 'whatever $R$ is'.

3. Section 2. In (4), the authors emphasize that $R^{L}$ is a non smooth external data which is not a function of the conservative variables $(\rho, \rho U)$ : is this data a function of $x, t$ ? of other varying quantities? at this level, there is some lack of information, the only example given afterwards is $\rho R^{L}=\pi(\rho)$, and in appendix E, some noise is added. Then, can (4) really be written in conservative form with $F_{\phi}\left(W_{\phi}\right)$ if $R^{L}$ is indeed a non smooth external data which is not a function of the conservative variables $W_{\phi}=(\rho, \rho U)^{T}$ ?

In fact, $R_{i j}^{L}, \forall i, j$ is a function of $(x, t)$ as emphasized on page 1 line 9 . In the present version of the paper, it is also underlined further (see p. 3, line 1) as "It is necessary to recall first that, since kinetic tensor $\mathbf{R}^{\mathbf{L}}(\mathbf{t}, \mathbf{x})$ is non-smooth external data which, in practical coupled computations with the Lagrangian code, is not a function of variables $(\rho, \rho \mathbf{U})$, we are formally interested in finding "discontinuous" solutions of the problem". 
Then, in section 3, it is in fact an error in writting $F_{\phi}\left(W_{\phi}\right)$ in formula (31) and below. Instead, it must be $F_{\phi}\left(W_{\phi}, R^{L}\right)$, and it has been modified accordingly (see eqs. (31) - (33)).

4. Section 2. page 3, line 3, 'smooth solution on a coarse mesh': we are still at the PDE level, before any approximation, why is any mesh mentioned? This remark should be clarified.

This expression has been removed and the idea has been reformulated as follows: "...we are formally interested in finding "discontinuous" solutions of the problem. This may happen even if $R^{L}(t, x)=c s t>0$."

5. Section 2. page 3, in (C4), there is a reference to (A1) which is not yet defined: this should be made more precise by introducing some term such as 'below', or 'in next section', or 'in Proposition 2'...

The remark has been taken into account as follows: (C4) The relaxation system should be close enough to (A1) (introduced in [26, 27] and recalled below in sec. 2.2) that represents a relevant physical choice.

6. Section 2. page 4, around (9), unify notation $A(Z), A(Z)$.

This has been modified.

7. Section 2. Proposition 2 should be written in a more rigorous way: there should not be a reference to [23], moreover, (A1) and (A2) should be defined as assumptions on $\Psi$, in the present manuscript, they are whole assertions. There might be a reference to system (7), and the sentence 'in order to ensure...' should also be put after the proposition. Then the sentence in Proposition 2 could be something like: 'Assuming $\Psi$ is given by (A1), system (7) admits two genuinely non linear fields and...', and 'Assuming $\Psi$ is given by (A2), system (7) admits three linearly degenerate fields...' or any sentence where the assumptions and the conclusions are clearly set.

Proposition 2 has changed to property and has been reformulated following the remarks made by the reviewer:

A natural choice for $\Psi$ arising from $R_{i j}$ model in one-dimensional space was given in [27]: $\Psi=3 \rho R$. This is referred to as approach (A1).

Property 2.

(A1): If we assume that $\Psi=3 \rho R$, then system (9) admits two GNL fields and one LD field;

(A2): If we assume that $\Psi=a_{0}^{2} \vartheta$ with constant $a_{0} \in \mathbb{R}_{+}^{*}$, then system (9) admits three LD fields.

Expression "In order to ensure the link with the expression in (A1), in (A2) we fix $a_{0}=\sqrt{3 R_{0} \rho_{0}^{2}}$ (see Appendix A (part II)" has been put after the "proof".

8. Section 2. Remark 2: $\partial_{x} U \in L^{\infty}\left(\mathbb{R}_{x} \times[0, T]\right)$ excludes discontinuous $U$, what if $U$ is discontinuous?

If $U$ is discontinuous, the solution of the associated Riemann problem is necessary to conclude. 
9. Section 2. At the beginning of Section 2.3, the Riemann problem is defined for the homogeneous part of system (7) which is in conservative form while (13) is solved in nonconservative variable $Z=(\rho, U, \rho R)^{T}$, as in Proposition 3. Then in section 3.1, the scheme is written in conservative variable $W=(\rho, \rho U, \rho R)^{T}$, refering to Proposition 3. That might be clarified a little.

We introduce variable $Z$ to simplify some calculations presented in section 2 . Then, in section 3.1 the scheme is written in conservative variable $W$.

10. Section 2. page 6, in Proposition 3 and in Figure 1, the eigenvalues $\lambda_{k}$ are not constant, even if each is a k-Riemann invariant, they depend on the data $Z_{l}, Z_{r}$, thus if $\lambda_{k}$ is a specific notation, it should be introduced somewhere, at least in the proof. A similar remark holds for Figure 2 where it should be $\lambda_{k, i+1 / 2}$.

This has been corrected following the remark of the reviewer (see fig. 1, 2).

11. Section 3. Why do the authors introduce a notation $\phi$ in (31)?

In section 3, the subscript $\phi$ has been introduced in order to make the difference between the solution $W_{\phi}=(\rho, \rho U)^{t}$ of original system (4) ( $\phi$ like 'physical'), and the solution $W=(\rho, \rho U, \rho R)^{t}$ of extended system (7), where this subscript is intentionally omitted.

12. Section 3. If remark 7 needs a proof, it is more a proposition than a simple remark.

Since this remark presents a well-known result, we prefer to keep it further as a simple remark rather than a proposition. The former proof has been removed.

13. Conclusion. (A1) and (A2) are now referred to as 'schemes', again, this is not correctly written.

This has been modified, the word 'scheme' has been replaced by 'approach' or 'method'.

14. Many articles 'the' or 'a' are still missing, for instance, in proposition 1, it should be the 'homogeneous system corresponding to'...), there are many other examples; also write Whitham's condition.

The paper has been reread on the whole, we have taken into account all the remarks made by the reviewer and many other corrections have been made. 